\title{
Scalable Modeling and Solution of Stochastic Multiobjective Optimization Problems
}

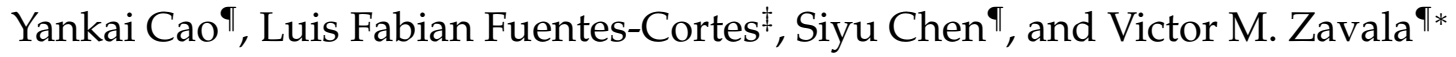 \\ "Department of Chemical and Biological Engineering \\ University of Wisconsin-Madison, 1415 Engineering Dr, Madison, WI 53706, USA \\ ${ }^{\ddagger}$ Department of Chemical Engineering \\ Universidad Michoacana de San Nicolas de Hidalgo, Morelia, Michoacan, Mexico, 58060
}

\begin{abstract}
We present a scalable computing framework for the solution stochastic multiobjective optimization problems. The proposed framework uses a nested conditional value-at-risk (nCVaR) objective to find compromise solutions among conflicting random objectives. We prove that the associated $\mathrm{nCVaR}$ minimization problem can be cast as a standard stochastic programming problem with expected value (linking) constraints. We also show that these problems can be implemented in a modular and compact manner using PLASMO (a Julia-based structured modeling framework) and can be solved efficiently using PIPS-NLP (a parallel nonlinear solver). We apply the framework to a CHP design study in which we seek to find compromise solutions that trade-off cost, water, and emissions in the face of uncertainty in electricity and water demands.
\end{abstract}

Keywords: large scale; optimization; stochastic; multiobjective; CVaR

\section{Motivation}

Stochastic multiobjective optimization (SMOO) problems trade-off multiple random objectives, multiple statistics (metrics) of a single random objective, or combinations of both (e.g., expected cost vs. expected environmental impact, expected cost vs. worst-case cost, expected cost vs. worst-case environmental impact) [1,19]. These types of problems also arise in multi-stakeholder decisionmaking in which a population of stakeholders seek to balance their conflicting objectives and priorities (e.g., attitudes and perceptions towards risk). One special case of SMOO is deterministic multiobjective optimization (MOO), for which general formulations and computational frameworks are well-established [35, 41, 22]. SMOO formulations have a a wide range of applications that include finance [2], energy systems [9], chemical processes [43, 37, 31], transportation logistics [21], facility location [16, 14], manufacturing and production planning [42], supply chain management [40], telecommunication, health care management [3,5], budget allocation [23], and project management [20].

*Corresponding Author: victor.zavala@wisc.edu 
SMOO problems present several interesting technical challenges. In particular, SMOO problems are inherently ill-posed, in the sense that no unique solution exists. Consequently, a decision-maker needs to select appropriate metrics to identify efficient solutions (i.e., in the sense of Pareto or stochastic dominance) $[19,24]$. SMOO problems are also inherently high-dimensional because they capture multiple realizations of random data and potentially many objectives. Consequently, in many applications it is impractical to compute the entire Pareto set. The most popular approach compute solutions for SMOO problems is scalarization. Here, the idea is to create a weighted combination of the random objectives and then minimize a summarizing statistic (e.g., expected value or a coherent risk metric) of the weighted objective function. Another approach is to explicitly enforce stochastic dominance over a predefined target distribution of the weighted objective [12] and for all the objectives $[13,4]$. The stochastic dominance approach, while more flexible, is also computationally more demanding as it requires specialized mixed-integer reformulations. Because of this, the scalarization approach is often preferred but this requires experimentation with different metrics to shape the objective distributions.

In this work, we present a computational framework to solve high-dimensional continuous and nonconvex SMOO problems using a specialized scalarization technique. Our framework uses a general metric, that we call the nested conditional value at risk (nCVaR), to compute compromise solutions for such problems. This metric extracts subsets of the largest objectives and realizations that we seek to minimize. We show that the framework allows us to capture a wide range of scalarization metrics of interest and has expected value and worst-case metrics as extreme cases. We also show that the $\mathrm{nCVaR}$ minimization problem can be cast as standard stochastic programming problem with expected value constraints. This allows us to use linear algebra decomposition strategies to accelerate solutions [10]. We also illustrate how to model these problems in a compact manner using PLASMO [25], which is a Julia-based graph modeling framework that facilitates the expression and manipulation of structured problems.

The paper is structured as follows. In Section 2 we present the SMOO setting and introduce the nested CVaR metric and reformulations. In Section 3 we present a scalable modeling a solution framework for SMOO. In Section 4 we illustrate the developments using a design case study for a combined heat and power plant that seeks to trade-off cost, emissions, and water in the face of uncertainty in electricity and water demands. The paper closes with final remarks and directions of future work.

\section{Stochastic Multiobjective Optimization Problems}

In this section, we state the SMOO problem under study and present the nested CVaR metric to compute compromise solutions for this problem. 


\subsection{Setting and Basic Definitions}

We are interested in finding solutions to the SMOO problem:

$$
\min _{x \in \mathcal{X}}\left\{\mathbf{F}_{0,0}(x), \mathbf{F}_{0,1}(x), \ldots, \mathbf{F}_{0, m-1}(x), \mathbf{F}_{1,0}(x), \mathbf{F}_{1,1}(x), \ldots, \mathbf{F}_{1, m-1}(x), \ldots, \mathbf{F}_{n-1, m-1}(x)\right\}
$$

Where $x \in \mathcal{X} \subseteq \mathbb{R}^{n_{x}}$ are the decision variables, $\mathbf{F}_{i, j}: \mathcal{X} \rightarrow \mathbb{R}$ are nonconvex and continuously differentiable objective functions, $\mathcal{X}:=\{x \mid h(x) \geq 0\}$ is the feasible set (with continuously differentiable but possibly non-convex constraints $h: \mathbb{R}^{n_{x}} \rightarrow \mathbb{R}^{n_{\nu}}$ and with associated dual variables $\left.\nu \in \mathbb{R}^{n_{\nu}}\right)^{1}$. The index $i \in \mathcal{O}:=\{0, \ldots, n-1\}$ is the objective index and $j \in \mathcal{S}:=\{0, \ldots, m-1\}$ is the scenario index. Each scenarios $j \in \mathcal{S}$ can be used to represent realizations of the objectives of the form $\mathbf{F}_{i, j}(x):=p_{j} \mathbf{F}_{i}\left(x, \xi_{j}\right)$ where $\xi_{j} \in \mathbb{R}^{n_{\xi}}$ is a realization of a random variable with probability $p_{j} \in \mathbb{R}_{+}$. Objectives can also be of the form $\mathbf{F}_{i, j}(x):=w_{i} \mathbf{F}_{i, j}(x)$ where $w_{i} \in \mathbb{R}_{+}$are weights used to prioritize different objectives and scenarios. We thus note that probabilities and weights can be interpreted in a similar way to create the more general form $\mathbf{F}_{i, j}(x):=w_{i, j} \mathbf{F}_{i, j}(x)$. This setting can thus be used to capture multiple objectives (e.g., economic,environmental,social) and/or priorities from different stakeholders (e.g., in a competitive setting) [24].

For convenience, we define the objective matrix $\mathbf{F}(x) \in \mathbb{R}^{n \times m}$ with entries $\mathbf{F}_{i, j}(x)$. We use the notation $\mathbf{F}_{i,:} \in \mathbb{R}^{m}$ to denote the i-th row of matrix $\mathbf{F}$ and $\mathbf{F}_{:, j} \in \mathbb{R}^{n}$ to denote the $\mathrm{j}$-th column of matrix $\mathbf{F}$. To highlight differences between scenario and objective spaces, we will refer to $\frac{1}{n} \sum_{i \in \mathcal{O}} \mathbf{F}_{i, j}$ (for a given $j \in \mathcal{S}$ ) as the average objective in the objective space. In addition, we will refer to $\frac{1}{m} \sum_{j \in \mathcal{S}} \mathbf{F}_{i, j}$ (for a fixed $i \in \mathcal{O}$ ) as the mean objective in the scenario space. Similarly, we refer to $\max _{i \in \mathcal{O}} \mathbf{F}_{i, j}$ as the largest objective and to $\max _{j \in \mathcal{S}} \mathbf{F}_{i, j}$ as the worst-case objective.

In a multiobjective setting it is often desired to scale the objectives. This can be done by using the so-called utopia solutions, which are computed from the individual problems:

$$
\mathbf{F}_{i, j}^{*}:=\min _{x \in \mathcal{X}} \mathbf{F}_{i, j}(x), \quad(i, j) \in \mathcal{O} \times \mathcal{S}
$$

With this, we can scale the objectives as $\mathbf{F}_{i, j}(x) \leftarrow \mathbf{F}(x) / \mathbf{F}_{i, j}^{*}$ (provided that $\mathbf{F}_{i, j}^{*}>0$ ). Many other scaling alternatives as possible (e.g., linear scaling using utopia and nadir points) [34, 14].

The objectives $\mathbf{F}_{i, j}(x)$ are usually conflicting and thus SMOO does not have a well-defined solution. We thus seek to find compromise solutions that balance all objectives and in all scenarios. This

\footnotetext{
${ }^{1}$ We generalize this formulation to consider recourse variables in Section 3.
} 
can also be done in multiple ways; consider, for instance, the following problems:

$$
\begin{aligned}
& \min _{x \in \mathcal{X}} \frac{1}{n \cdot m} \sum_{(i, j) \in \mathcal{O} \times \mathcal{S}} \mathbf{F}_{i, j}(x) \\
& \min _{x \in \mathcal{X}} \frac{1}{n} \sum_{i \in \mathcal{O}} \max _{j \in \mathcal{S}} \mathbf{F}_{i, j}(x) \\
& \min _{x \in \mathcal{X}} \frac{1}{m} \sum_{j \in \mathcal{S}} \max _{i \in \mathcal{O}} \mathbf{F}_{i, j}(x) \\
& \min _{x \in \mathcal{X}} \max _{j \in \mathcal{S}} \frac{1}{n} \sum_{i \in \mathcal{O}} \mathbf{F}_{i, j}(x) \\
& \min _{x \in \mathcal{X}} \max _{i \in \mathcal{O}} \frac{1}{m} \sum_{j \in \mathcal{S}} \mathbf{F}_{i, j}(x) \\
& \min _{x \in \mathcal{X}} \max _{(i, j) \in \mathcal{O} \times \mathcal{S}} \mathbf{F}_{i, j}(x)
\end{aligned}
$$

Problem (2.3a) minimizes the mean of the average objectives; (2.3f) minimizes the largest worst-case objective; (2.3b) minimizes the mean of the worst-case objectives; (2.3c) minimizes the average of the largest objectives, and so on. The solutions of these problems have different interpretations that might desirable in different decision-making contexts. To highlight how different interpretations arise, we consider the following problem:

$$
\mathbf{F}_{:, j}:=\min _{x \in \mathcal{X}} \frac{1}{n} \sum_{i \in \mathcal{O}} \mathbf{F}_{i, j}(x), \quad j \in \mathcal{S} .
$$

The solution of this problem minimizes the average objective for a given scenario $j \in \mathcal{S}$. This solution can also be interpreted as minimum distance solution (under an $\ell_{1}$ norm) to the utopia point $\mathbf{F}_{:, j}^{*}$ (the vector $\mathbf{F}_{:, j}$ contains the coordinates of the mimimum-distance point) [48]. One can prove that the minimum distance solution is a Pareto solution associated to the scenario $j \in \mathcal{S}$ (i.e., each scenario has an associated Pareto set). Consequently, by solving (2.3a), we find a solution that minimizes the mean of the of the average-distance solutions to the utopia point and one can prove that this solution is Pareto optimal in the combined space of the objectives and scenarios. This solution can also be interpreted as the minimization of an entry-wise $\ell_{1}$ matrix norm $\|\mathbf{F}(x)\|_{1}$. Following this line of thought, the solution of the problem

$$
\mathbf{F}_{:, j}:=\min _{x \in \mathcal{X}} \max _{i \in \mathcal{O}} \mathbf{F}_{i, j}(x), \quad j \in \mathcal{S}
$$

gives the minimum distance solution (under the $\ell_{\infty}$ norm) to the utopia point and the vector $\mathbf{F}_{:, j}$ contains the coordinates of the minimum-distance point [48]. This solution can also be interpreted as that of finding the minimum largest objective for a given scenario $j \in \mathcal{S}$. Consequently, by solving (2.3c) we find a solution that minimizes the average of the $\ell_{\infty}$-distance solutions to the utopia point, while by solving (2.3f) we find a solution that minimizes the worst-case of the $\ell_{\infty}$-distance solutions to the utopia point. This can also be interpreted as the minimization of an entry-wise $\ell_{\infty}$ matrix norm $\|\mathbf{F}(x)\|_{\infty}$. 


\subsection{Nested CVaR Metric}

We use the conditional value at risk $(\mathrm{CVaR})$ to create a general framework to compute a wide range of compromise solutions for SMOO. For clarity in the presentation, we define CVaR using finitedimensional vector spaces (which also have geometric interpretations). For a given vector $\mathbf{v} \in \mathbb{R}^{n_{v}}$ with entries given by realizations $v_{\ell}, \ell \in\left\{1, \ldots, n_{v}\right\}$, we denote its CVaR with probability level $\alpha \in$ $[0,1]$ as $\langle\langle\mathbf{v}\rangle\rangle_{\alpha}$. Assume now (without loss of generality) that the entries of $\mathbf{v}$ are arranged as $v_{1} \leq v_{2} \leq$ $\cdots \leq v_{n_{v}}$. For a fixed $k \in\left\{0, \ldots, n_{v}-1\right\}$ with the probability level $\alpha(k):=k / n_{v}, \mathrm{CVaR}$ is computed as:

$$
\langle\langle\mathbf{v}\rangle\rangle_{\alpha(k)}=\frac{1}{n_{v}-k} \sum_{\ell=k+1}^{n_{v}} v_{\ell} .
$$

In other words, CVaR with probability level $\alpha(k)$ is the average of the $n_{v}-k$ largest entries of $\mathbf{v}$. Note also that $\langle\langle\mathbf{v}\rangle\rangle_{\alpha(0)}=\frac{1}{n_{v}} \sum_{\ell=1}^{n_{v}} \mathbf{v}_{\ell}$ and $\langle\langle\mathbf{v}\rangle\rangle_{\alpha\left(n_{v}-1\right)}=\max _{\ell \in\left\{1, \ldots, n_{v}\right\}}\left\{\mathbf{v}_{\ell}\right\}$. It is also well-known that CVaR can be computed by solving the optimization problem:

$$
\langle\langle\mathbf{v}\rangle\rangle_{\alpha(k)}=\min _{t} t+\frac{1}{(1-\alpha(k)) \cdot n_{v}} \sum_{\ell=1}^{n_{v}}\left[v_{\ell}-t\right]_{+},
$$

where $\left[v_{\ell}\right]_{+}=\max \left\{0, v_{\ell}\right\}$ and $k \in\left\{0, \ldots, n_{v}-1\right\}$ and the value of $t$ that minimizes the right-hand side of (2.7) is the value-at-risk [38]. We highlight that we can compute CVaR for a non-integer $k$ by using a weighted sum of the nearest integer values. The interested reader can refer to [38, 14] for more details. In the following, we drop the dependence of $\alpha(k)$ on $k$ and simply use $\alpha$. Moreover, we set $\langle\langle\mathbf{v}\rangle\rangle_{\alpha=1}=\langle\langle\mathbf{v}\rangle\rangle_{\alpha\left(n_{v}-1\right)}$ and use the notation $\alpha=0$ to imply that $k=0$ and $\alpha=1$ to imply that $k=n_{v}-1$. Moreover, the notation $\alpha=1$ indicates that (2.7) is solved with $\alpha\left(n_{v}-1\right)$.

We now define the following quantities:

$$
\begin{gathered}
\hat{\mathbf{F}}_{\alpha_{\mathcal{O}}}^{j}(x):=\left\langle\left\langle\mathbf{F}_{:, j}\right\rangle\right\rangle_{\alpha_{\mathcal{O}}}, j \in \mathcal{S} \\
\hat{\mathbf{F}}_{\alpha_{\mathcal{S}}}^{i}(x):=\left\langle\left\langle\mathbf{F}_{i,:}\right\rangle\right\rangle_{\alpha_{\mathcal{S}}}, i \in \mathcal{O}
\end{gathered}
$$

where $\alpha_{\mathcal{O}}$ and $\alpha_{\mathcal{S}}$ are probability levels for vectors with cardinality $n=|\mathcal{O}|$ and $m=|\mathcal{S}|$, respectively. We also define vectors $\hat{\mathbf{F}}_{\alpha_{\mathcal{O}}}(x) \in \mathbb{R}^{m}$ with entries $\hat{\mathbf{F}}_{\alpha_{\mathcal{O}}}^{j}(x)$ and $\hat{\mathbf{F}}_{\alpha_{\mathcal{S}}}(x) \in \mathbb{R}^{n}$ with entries $\hat{\mathbf{F}}_{\alpha_{\mathcal{S}}}^{i}(x)$.

From these definitions and the properties of $\mathrm{CVaR}$ we can immediately establish that:

$$
\begin{aligned}
\hat{\mathbf{F}}_{\alpha_{\mathcal{O}}=0}^{j}(x) & =\frac{1}{n} \sum_{i \in \mathcal{O}} \mathbf{F}_{i, j}(x), j \in \mathcal{S} \\
\hat{\mathbf{F}}_{\alpha_{\mathcal{S}}=0}^{i}(x) & =\frac{1}{m} \sum_{j \in \mathcal{S}} \mathbf{F}_{i, j}(x), i \in \mathcal{O} \\
\hat{\mathbf{F}}_{\alpha_{\mathcal{O}}=1}^{j}(x) & =\max _{i \in \mathcal{O}}\left\{\mathbf{F}_{i, j}(x)\right\}, j \in \mathcal{S} \\
\hat{\mathbf{F}}_{\alpha_{\mathcal{S}}=1}^{i}(x) & =\max _{j \in \mathcal{S}}\left\{\mathbf{F}_{i, j}(x)\right\}, i \in \mathcal{O} .
\end{aligned}
$$

We use these quantities to define the nested CVaR (nCVaR) metrics:

$$
\begin{aligned}
\langle\langle\mathbf{F}(x))\rangle_{\alpha_{\mathcal{O}}, \alpha_{\mathcal{S}}} & :=\left\langle\left\langle\hat{\mathbf{F}}_{\alpha_{\mathcal{S}}}(x)\right\rangle\right\rangle_{\alpha_{\mathcal{O}}} \\
\langle\langle\mathbf{F}(x))\rangle_{\alpha_{\mathcal{S}}, \alpha_{\mathcal{O}}} & :=\left\langle\left\langle\hat{\mathbf{F}}_{\alpha_{\mathcal{O}}}(x)\right\rangle\right\rangle_{\alpha_{\mathcal{S}}} .
\end{aligned}
$$


The $\mathrm{nCVaR}$ metrics are used to state the optimization problems:

$$
\begin{aligned}
& \min _{x \in \mathcal{X}}\langle\langle\mathbf{F}(x)\rangle\rangle_{\alpha_{\mathcal{O}}, \alpha_{\mathcal{S}}} \\
& \min _{x \in \mathcal{X}}\langle\langle\mathbf{F}(x)\rangle\rangle_{\alpha_{\mathcal{S}}, \alpha_{\mathcal{O}}}
\end{aligned}
$$

which we will use to compute compromise solutions for SMOO. Nested CVaR is a generalized metric that can be used to compute families of compromise solutions with different interpretations and has problems (2.3) as limiting cases. For instance, we have that:

$$
\begin{aligned}
\langle\langle\mathbf{F}(x))\rangle_{\alpha_{\mathcal{O}}=0, \alpha_{\mathcal{S}}=0} & =\langle\langle\mathbf{F}(x)\rangle\rangle_{\alpha_{\mathcal{S}}=0, \alpha_{\mathcal{O}}=0}=\frac{1}{n \cdot m} \sum_{(i, j) \in \mathcal{O} \times \mathcal{S}} \mathbf{F}_{i, j}(x) \\
\langle\langle\mathbf{F}(x))\rangle_{\alpha_{\mathcal{O}}=1, \alpha_{\mathcal{S}}=1} & =\langle\langle\mathbf{F}(x)\rangle\rangle_{\alpha_{\mathcal{S}}=1, \alpha_{\mathcal{O}}=1}=\max _{(i, j) \in \mathcal{O} \times \mathcal{S}} \mathbf{F}_{i, j}(x) \\
\langle\langle\mathbf{F}(x)\rangle\rangle_{\alpha_{\mathcal{O}}=0, \alpha_{\mathcal{S}}=1} & =\frac{1}{n} \sum_{i \in \mathcal{O}} \max _{j \in \mathcal{S}} \mathbf{F}_{i, j}(x) \\
\langle\langle\mathbf{F}(x)\rangle\rangle_{\alpha_{\mathcal{O}}=1, \alpha_{\mathcal{S}}=0} & =\max _{i \in \mathcal{O}} \frac{1}{m} \sum_{j \in \mathcal{S}} \mathbf{F}_{i, j}(x) \\
\langle\langle\mathbf{F}(x)\rangle\rangle_{\alpha_{\mathcal{S}}=0, \alpha_{O}=1} & =\frac{1}{m} \sum_{j \in \mathcal{S}} \max _{i \in \mathcal{O}} \mathbf{F}_{i, j}(x) \\
\langle\langle\mathbf{F}(x))\rangle_{\alpha_{\mathcal{S}}=1, \alpha_{O}=0} & =\max _{j \in \mathcal{S}} \frac{1}{n} \sum_{i \in \mathcal{O}} \mathbf{F}_{i, j}(x) .
\end{aligned}
$$

The $\mathrm{nCVaR}$ metric can also be used to trade-off $\mathrm{CVaR}$ metrics for different objectives. For instance, we can trade-off CVaR for an economic metric, CVaR for environmental metric, and CVaR for a social metric. In particular, consider the case in which we want to minimize the average of the $90 \%$ probability CVaR metrics for the objectives. This problem can be formulated as (2.11a) with $\alpha_{\mathcal{O}}=0$ and $\alpha_{\mathcal{S}}=0.9$ and is equivalent to:

$$
\min _{x \in \mathcal{X}} \frac{1}{n} \sum_{i \in \mathcal{O}}\left\langle\left\langle\mathbf{F}_{i,:}(x)\right\rangle\right\rangle_{\alpha_{\mathcal{S}}}
$$

or, equivalently,

$$
\min _{x \in \mathcal{X}} \frac{1}{n} \sum_{i \in \mathcal{O}}\left(\min _{t_{i}}\left\{t_{i}+\frac{1}{\left(1-\alpha_{\mathcal{S}}\right) \cdot m} \sum_{j \in \mathcal{S}}\left[\mathbf{F}_{i, j}(x)-t_{i}\right]_{+}\right\}\right) .
$$

Similarly, we can minimize the largest of the $90 \%$-probability CVaR metrics for the objectives by solving (2.11a) with $\alpha_{\mathcal{O}}=1$ and $\alpha_{\mathcal{S}}=0.9$. As another example, by minimizing (2.11a) with $\alpha_{\mathcal{O}}=$ $0.9, \alpha_{\mathcal{S}}=0$ would minimize the largest $10 \%$ of the mean objectives and has the form:

$$
\min _{x \in \mathcal{X}}\left\langle\left\langle\frac{1}{m} \sum_{j \in \mathcal{S}} \mathbf{F}_{:, j}(x)\right\rangle\right\rangle_{\alpha_{\mathcal{O}}} .
$$

This situation arises when we seek to find a solution that finds a compromise between the objectives of multiple stakeholders (in this case $i \in \mathcal{O}$ represents a stakeholder). We also note that since $\mathrm{CvaR}$ is a vector norm [38], it can be verified that the nCVaR metric can be interpreted as a matrix norm (provided that $\mathbf{F}_{i, j}(x) \geq 0$ for all $x \in \mathcal{X}$ ). 


\subsection{Nested CVaR Reformulations}

By combining (2.7), (2.8a), and (2.10a) we can formulate (2.11a) as:

$$
\min _{x \in \mathcal{X}, z} z+\frac{1}{n\left(1-\alpha_{\mathcal{O}}\right)} \sum_{i \in \mathcal{O}}\left[\min _{t_{i}}\left\{t_{i}+\frac{1}{m\left(1-\alpha_{\mathcal{S}}\right)} \sum_{j \in \mathcal{S}}\left[\mathbf{F}_{i, j}(x)-t_{i}\right]_{+}\right\}-z\right]_{+} .
$$

This problem can also be reformulated as:

$$
\begin{aligned}
\min _{x \in \mathcal{X}, z, \hat{\mathbf{F}}_{\alpha_{\mathcal{S}}}^{i}} \varphi\left(z, \hat{\mathbf{F}}_{\alpha_{\mathcal{S}}}\right) & \\
\text { s.t. } & \hat{\mathbf{F}}_{\alpha_{\mathcal{S}}}^{i}=\min _{t_{i}}\left\{t_{i}+\frac{1}{m\left(1-\alpha_{\mathcal{S}}\right)} \sum_{j \in \mathcal{S}}\left[\mathbf{F}_{i, j}(x)-t_{i}\right]_{+}\right\}, i \in \mathcal{O}
\end{aligned}
$$

where $\varphi\left(z, \hat{\mathbf{F}}_{\alpha_{\mathcal{S}}}\right):=z+\frac{1}{n\left(1-\alpha_{\mathcal{O}}\right)} \sum_{i \in \mathcal{O}}\left[\hat{\mathbf{F}}_{\alpha_{\mathcal{S}}}^{i}-z\right]_{+}$. Problems (2.16) and (A) cannot be solved directly using off-the-shelf NLP solvers because they include inner min and max operators. We now proceed to show that we reformulate (A) as a standard NLP. We first prove that (A) can be reformulated as:

$$
\begin{aligned}
\min _{x \in \mathcal{X}, z, \overline{\mathbf{F}}_{\alpha_{\mathcal{S}}}^{i}, t_{i}} & \varphi\left(z, \overline{\mathbf{F}}_{\alpha_{\mathcal{S}}}\right) \\
\text { s.t. } & \overline{\mathbf{F}}_{\alpha_{\mathcal{S}}}^{i}=t_{i}+\frac{1}{m\left(1-\alpha_{\mathcal{S}}\right)} \sum_{j \in \mathcal{S}}\left[\mathbf{F}_{i, j}(x)-t_{i}\right]_{+}, i \in \mathcal{O} .
\end{aligned}
$$

We define an optimal solution of (A) as $\left(x^{A}, z^{A}, \hat{\mathbf{F}}_{\alpha_{\mathcal{S}}}^{A}, t^{A}\right)$ where $t^{A} \in \mathbb{R}^{n}$ is a vector with entries $t_{i}^{A}$. We define the associated objective value of (A) as $v^{A}:=\varphi\left(z^{A}, \hat{\mathbf{F}}_{\alpha_{\mathcal{S}}}^{A}\right)$. We also define an optimal solution of (B) as $\left(x^{B}, z^{B}, \overline{\mathbf{F}}_{\alpha_{\mathcal{S}}}^{B}, t^{B}\right)$ and the corresponding objective value as $v^{B}:=\varphi\left(z^{B}, \overline{\mathbf{F}}_{\alpha_{\mathcal{S}}}^{B}\right)$.

Theorem 1 An optimal solution of (B) is an optimal solution of (A).

Proof: It suffices to show that $v^{A}=v^{B}$. It is straightforward to see that $\left(x^{A}, z^{A}, \hat{\mathbf{F}}_{\alpha_{\mathcal{S}}}^{i, A}, t^{A}\right)$ is a feasible solution to (B) and the objective function of (B) evaluated at $\left(x^{A}, z^{A}, \hat{\mathbf{F}}_{\alpha_{\mathcal{S}}}^{i, A}, t^{A}\right)$ is $\varphi\left(z^{A}, \hat{\mathbf{F}}_{\alpha_{\mathcal{S}}}^{i, A}\right)=v^{A}$. We thus have that $v^{B} \leq v^{A}$. If we now define $\hat{\mathbf{F}}_{\alpha_{\mathcal{S}}}^{i, B}:=\min _{t_{i}}\left\{t_{i}+\frac{1}{m\left(1-\alpha_{\mathcal{S}}\right)} \sum_{j \in \mathcal{S}}\left[\mathbf{F}_{i, j}\left(x^{B}\right)-t_{i}\right]_{+}\right\}$we note that $\left(x^{B}, z^{B}, \hat{\mathbf{F}}_{\alpha_{\mathcal{S}}}^{B}\right)$ is a feasible solution to (A). Moreover, because $\hat{\mathbf{F}}_{\alpha_{\mathcal{S}}}^{i, B} \leq \overline{\mathbf{F}}_{\alpha_{\mathcal{S}}}^{i, B}$, the objective value of (A) evaluated at the feasible solution $\left(x^{B}, z^{B}, \hat{\mathbf{F}}_{\alpha_{\mathcal{S}}}^{B}\right)$ satisfies

$$
\begin{aligned}
v^{A} & \leq \varphi\left(z^{B}, \hat{\mathbf{F}}_{\alpha_{\mathcal{S}}}^{B}\right) \\
& =z^{B}+\frac{1}{n\left(1-\alpha_{\mathcal{O}}\right)} \sum_{i \in \mathcal{O}}\left[\hat{\mathbf{F}}_{\alpha_{\mathcal{S}}}^{i, B}-z^{B}\right]_{+} \\
& \leq z^{B}+\frac{1}{n\left(1-\alpha_{\mathcal{O}}\right)} \sum_{i \in \mathcal{O}}\left[\overline{\mathbf{F}}_{\alpha_{\mathcal{S}}}^{i, B}-z^{B}\right]_{+} \\
& =\varphi\left(z^{B}, \overline{\mathbf{F}}_{\alpha_{\mathcal{S}}}^{B}\right) \\
& =v^{B} .
\end{aligned}
$$


Therefore, we also have that $v^{A} \leq v^{B}$ and the proof is complete.

Theorem 1 implies that problem (2.16) is equivalent to:

$$
\min _{x \in \mathcal{X}, z, t_{i}} z+\frac{1}{n\left(1-\alpha_{\mathcal{O}}\right)} \sum_{i \in \mathcal{O}}\left[t_{i}+\frac{1}{m\left(1-\alpha_{\mathcal{S}}\right)} \sum_{j \in \mathcal{S}}\left[\mathbf{F}_{i, j}(x)-t_{i}\right]_{+}-z\right]_{+} .
$$

In other words, the inner min operator can be factored out. To avoid the use of the non-smooth max functions, we now show that problem (B) can be reformulated as the standard nonlinear programming (NLP) problem:

$$
\begin{array}{rl}
\min _{x \in \mathcal{X}, z, \tilde{\mathbf{F}}_{\alpha_{\mathcal{S}}}^{i}, t_{i}, \eta_{i}, \mu_{i, j}} & z+\frac{1}{n\left(1-\alpha_{\mathcal{O}}\right)} \sum_{i \in \mathcal{O}} \eta_{i} \\
\text { s.t. } & \eta_{i} \geq 0, \quad i \in \mathcal{O} \\
& \eta_{i} \geq \tilde{\mathbf{F}}_{\alpha_{\mathcal{S}}}^{i}-z, \quad i \in \mathcal{O} \\
\tilde{\mathbf{F}}_{\alpha_{\mathcal{S}}}^{i}=t_{i}+\frac{1}{m\left(1-\alpha_{\mathcal{S}}\right)} \sum_{j \in \mathcal{S}} \mu_{i, j}, \quad i \in \mathcal{O} \\
\mu_{i, j} \geq 0, \quad i \in \mathcal{O}, j \in \mathcal{S} \\
\mu_{i, j} \geq \mathbf{F}_{i, j}(x)-t_{i}, \quad i \in \mathcal{O}, j \in \mathcal{S} .
\end{array}
$$

We use $\left(x^{C}, z^{C}, \tilde{\mathbf{F}}_{\alpha_{\mathcal{S}}}^{C}, t^{C}, \eta^{C}, \mu^{C}\right)$ and $v^{C}$ to denote an optimal solution and corresponding objective value of $(C)$, respectively.

Theorem 2 An optimal solution of (C) is an optimal solution of (B).

Proof: It suffices to show that $v^{B}=v^{C}$. If we define $\eta_{i}^{B}:=\left[\overline{\mathbf{F}}_{\alpha_{\mathcal{S}}}^{i, B}-z^{B}\right]_{+}$and $\mu_{i, j}^{B}:=\left[\mathbf{F}_{i, j}\left(x^{B}\right)-t_{i}\right]_{+}$ we have that $\left(x^{B}, z^{B}, \overline{\mathbf{F}}_{\alpha_{\mathcal{S}}}^{B}, t^{B}, \eta^{B}, \mu^{B}\right)$ is feasible to (C) and the objective function of (C) evaluated at $\left(x^{B}, z^{B}, \overline{\mathbf{F}}_{\alpha_{\mathcal{S}}}^{B}, t^{B}, \eta^{B}, \mu^{B}\right)$ is $z^{B}+\frac{1}{n\left(1-\alpha_{\mathcal{O}}\right)} \sum_{i \in \mathcal{O}} \eta_{i}^{B}=\varphi\left(z^{B}, \overline{\mathbf{F}}_{\alpha_{\mathcal{S}}}^{B}\right)=v^{B}$. We thus have that $v^{C} \leq v^{B}$.

If we now define $\overline{\mathbf{F}}_{\alpha_{\mathcal{S}}}^{i, C}:=t_{i}^{C}+\frac{1}{m\left(1-\alpha_{\mathcal{S}}\right)} \sum_{j \in \mathcal{S}}\left[\mathbf{F}_{i, j}\left(x^{C}\right)-t_{i}^{C}\right]_{+}$we have that $\left(x^{C}, z^{C}, \overline{\mathbf{F}}_{\alpha_{\mathcal{S}}}^{C}, t^{C}\right)$ is feasible to (B). This follows because $\mu_{i, j}^{C} \geq\left[\mathbf{F}_{i, j}\left(x^{C}\right)-t_{i}^{C}\right]_{+}$and

$$
\begin{aligned}
\overline{\mathbf{F}}_{\alpha_{\mathcal{S}}}^{i, C} & =t_{i}^{C}+\frac{1}{m\left(1-\alpha_{\mathcal{S}}\right)} \sum_{j \in \mathcal{S}}\left[\mathbf{F}_{i, j}\left(x^{C}\right)-t_{i}^{C}\right]_{+} \\
& \leq t_{i}^{C}+\frac{1}{m\left(1-\alpha_{\mathcal{S}}\right)} \sum_{j \in \mathcal{S}} \mu_{i, j}^{i, C} \\
& =\tilde{\mathbf{F}}_{\alpha_{\mathcal{S}}}^{i, C} .
\end{aligned}
$$

The objective function of (B) evaluated at $\left(x^{C}, z^{C}, \overline{\mathbf{F}}_{\alpha_{\mathcal{S}}}^{C}, t^{C}\right)$ is

$$
\begin{aligned}
z^{C}+\frac{1}{n\left(1-\alpha_{\mathcal{O}}\right)} \sum_{i \in \mathcal{O}}\left[\overline{\mathbf{F}}_{\alpha_{\mathcal{S}}}^{i, C}-z^{C}\right]_{+} & \leq z^{C}+\frac{1}{n\left(1-\alpha_{\mathcal{O}}\right)} \sum_{i \in \mathcal{O}}\left[\tilde{\mathbf{F}}_{\alpha_{\mathcal{S}}}^{i, C}-z^{C}\right]_{+} \\
& \leq z^{C}+\frac{1}{n\left(1-\alpha_{\mathcal{O}}\right)} \sum_{i \in \mathcal{O}} \eta_{i}^{C} \\
& =v^{C}
\end{aligned}
$$


Therefore, $v^{B} \leq v^{C}$.

Theorem 2 implies that problems (2.16) and (2.17) are equivalent to:

$$
\begin{array}{rl}
\min _{x \in \mathcal{X}, z, t_{i}, \mu_{i, j}} & z+\frac{1}{n\left(1-\alpha_{\mathcal{O}}\right)} \sum_{i \in \mathcal{O}}\left[t_{i}+\frac{1}{m\left(1-\alpha_{\mathcal{S}}\right)} \sum_{j \in \mathcal{S}} \mu_{i, j}-z\right]_{+} \\
\text {s.t. } & \mu_{i, j} \geq 0, \quad i \in \mathcal{O}, j \in \mathcal{S} \\
& \mu_{i, j} \geq \mathbf{F}_{i, j}(x)-t_{i}, \quad i \in \mathcal{O}, j \in \mathcal{S} .
\end{array}
$$

It is also easy to see that this problem is equivalent to:

$$
\begin{array}{rl}
\min _{x \in \mathcal{X}, z, t_{i}, \mu_{i, j}, \eta_{i}} & z+\frac{1}{n\left(1-\alpha_{\mathcal{O}}\right)} \sum_{i \in \mathcal{O}} \eta_{i} \\
\text { s.t. } & \eta_{i} \geq 0, \quad i \in \mathcal{O} \\
& \eta_{i} \geq t_{i}+\frac{1}{m\left(1-\alpha_{\mathcal{S}}\right)} \sum_{j \in \mathcal{S}} \mu_{i, j}-z, \quad i \in \mathcal{O} \\
& \mu_{i, j} \geq 0, \quad i \in \mathcal{O}, j \in \mathcal{S} \\
& \mu_{i, j} \geq \mathbf{F}_{i, j}(x)-t_{i}, \quad i \in \mathcal{O}, j \in \mathcal{S}
\end{array}
$$

and to problem (C). In other words, Theorem 2 indicates that the inner max operators can also be factored out to achieve the standard NLP representation (C).

Similar reformulations and properties can be derived for $(2.11 \mathrm{~b})$. In particular, we can reformulate (2.11b) as:

$$
\begin{array}{rl}
\min _{x \in \mathcal{X}, z, \tilde{\mathbf{F}}_{\alpha_{\mathcal{S}}}^{j}, t_{j}, \eta_{j}, \mu_{i, j}} & z+\frac{1}{m\left(1-\alpha_{\mathcal{S}}\right)} \sum_{j \in \mathcal{S}} \eta_{j} \\
\text { s.t. } & \eta_{j} \geq 0, \quad j \in \mathcal{S} \\
& \eta_{j} \geq \tilde{\mathbf{F}}_{\alpha_{\mathcal{S}}}^{j}-z, \quad j \in \mathcal{S} \\
& \tilde{\mathbf{F}}_{\alpha_{\mathcal{S}}}^{j}=t_{j}+\frac{1}{n\left(1-\alpha_{\mathcal{O}}\right)} \sum_{i \in \mathcal{O}} \mu_{i, j}, \quad j \in \mathcal{S} \\
& \mu_{i, j} \geq 0, \quad i \in \mathcal{O}, j \in \mathcal{S} \\
& \mu_{i, j} \geq \mathbf{F}_{i, j}(x)-t_{j}, \quad i \in \mathcal{O}, j \in \mathcal{S} .
\end{array}
$$

\subsection{Extensions}

The previous definitions and properties hold for problems with recourse variables and constraints. In particular, consider the problem:

$$
\min _{x \in \mathcal{X}, y_{i, j} \in \mathcal{Y}_{i, j}(x)}\left\langle\langle\mathbf{F}(x, y)\rangle_{\alpha_{\mathcal{O}}, \alpha_{\mathcal{S}}} .\right.
$$

Here, we note that we have generalized the concept of recourse in the sense that recourse variables can be used in the scenario and in the objective space. This can help us model multi-stakeholder 
settings, for instance, in which stakeholders have different sets of recourse variables. In a traditional SMOO setting, recourse variables are only introduced in the scenario space, to obtain a restricted problem of the form:

$$
\min _{x \in \mathcal{X}, y_{j} \in \mathcal{Y}_{j}(x)}\left\langle\langle\mathbf{F}(x, y)\rangle_{\alpha_{\mathcal{O}}, \alpha_{\mathcal{S}}}\right.
$$

If we consider feasible sets of the form $\mathcal{X}:=\{h(x) \geq 0\}$ and $\mathcal{Y}_{i, j}\left(x, y_{i, j}\right):=\left\{h_{i, j}\left(x, y_{i, j}\right) \geq 0\right\}$, problem (2.21) becomes an NLP of the form:

$$
\begin{array}{rl}
\min _{x, y_{i, j}, z, \tilde{\mathbf{F}}_{\alpha_{\mathcal{S}}}^{i}, t_{i}, \eta_{i}, \mu_{i, j}} & z+\frac{1}{n\left(1-\alpha_{\mathcal{O}}\right)} \sum_{i \in \mathcal{O}} \eta_{i} \\
\text { s.t. } & \eta_{i} \geq 0, \quad i \in \mathcal{O} \\
& \eta_{i} \geq \tilde{\mathbf{F}}_{\alpha_{\mathcal{S}}}^{i}-z, \quad i \in \mathcal{O} \\
& \tilde{\mathbf{F}}_{\alpha_{\mathcal{S}}}^{i}=t_{i}+\frac{1}{m\left(1-\alpha_{\mathcal{S}}\right)} \sum_{j \in \mathcal{S}} \mu_{i, j}, \quad i \in \mathcal{O} \\
& \mu_{i, j} \geq 0, \quad i \in \mathcal{O}, j \in \mathcal{S} \\
& \mu_{i, j} \geq \mathbf{F}_{i, j}\left(x, y_{i, j}\right)-t_{i}, \quad i \in \mathcal{O}, j \in \mathcal{S} \\
& h(x) \geq 0 \\
& h_{i, j}\left(x, y_{i, j}\right) \geq 0, \quad i \in \mathcal{O}, j \in \mathcal{S} .
\end{array}
$$

We also note that the nested $\mathrm{CVaR}$ metric can be easily adapted to handle the more general case in which we use different probability levels for each CVaR metric used in the scenario space (i.e., we use $\alpha_{\mathcal{S}}^{i}$ for $i \in \mathcal{O}$ ). For the example presented in (2.14), for instance, this would give a more general problem of the form:

$$
\min _{x \in \mathcal{X}} \frac{1}{n} \sum_{i \in \mathcal{O}}\left(\min _{t_{i}} t_{i}+\frac{1}{\left(1-\alpha_{\mathcal{S}}^{i}\right) \cdot m} \sum_{j \in \mathcal{S}}\left[\mathbf{F}_{i, j}(x)-t_{i}\right]_{+}\right) .
$$

This solution would thus minimize the average of the CVaR metrics but each CVaR metric would have its own probability level (e.g., $\alpha_{\mathcal{S}}^{0}=0, \alpha_{\mathcal{S}}^{1}=0.9, \ldots, \alpha_{\mathcal{S}}^{m-1}=1.0$ ). The same observations hold for the reverse case in which we can pick a different a different probability level for CVaR in the objective space $\left(\alpha_{\mathcal{O}}^{j}\right.$ for $j \in \mathcal{S}$ ). As can be seen, the proposed SMOO framework allows us to consider a wide range of problem classes.

\section{Scalable Modeling and Solution of SMOO Problems}

In this section we present a modeling and solution framework to address large-scale SMOO problems. In particular, we illustrate how to exploit linear algebra structures and how to create highly modular model implementations. 


\subsection{Problem Decomposition}

The SMOO problems can be cast as structured NLPs of the form:

$$
\begin{aligned}
& \min _{w_{0}, w_{p}} \phi_{0}\left(w_{0}\right)+\sum_{p \in \mathcal{P}} \phi_{p}\left(w_{0}, w_{p}\right) \\
& \text { s.t. } h_{0}\left(w_{0}\right) \geq 0 \quad\left(\nu_{0}\right) \\
& h_{p}\left(w_{0}, w_{p}\right) \geq 0, p \in \mathcal{P} \quad\left(\nu_{p}\right) \\
& \sum_{p \in \mathcal{P}}\left(\bar{\Pi}_{p} w_{0}+\underline{\Pi}_{p} w_{p}\right)=0 \quad(\lambda)
\end{aligned}
$$

Here, $w_{p}$ are the variables of the partition or scenario $p \in \mathcal{P}:=\{1,2, \ldots, P\}$ (recourse or second-stage variables) and $w_{0}$ are the coupling variables (first-stage variables). Functions $\phi_{p}(\cdot)$ and $h_{p}(\cdot)$ are the objective and constraints of partition $p \in \mathcal{P}$ and $\phi_{0}(\cdot)$ and $h_{0}(\cdot)$ are the counterparts of the first-stage. The dual variables of the first-stage and second-stage constraints are $\nu_{0}$ and $\nu_{p}$, respectively. We highlight that problem (3.25) is not a standard two-stage stochastic NLP due to the presence of the linking constraints (3.25d). These constraints have associated dual variables $\lambda$.

The linking constraints couple variables across scenarios. In our context, these constraints arise from the nested CVaR reformulations. For instance, note that constraint (2.23d) induces coupling of variables across scenarios. Under appropriate modifications, the NLP (3.25) can still be decomposed to enable parallel solutions. To explain this and without loss of generality, we ignore the inequality constraints in (3.25) and only highlight the linking constraints. Under such simplifications, the Lagrange function of (3.25) is given by:

$$
\mathcal{L}=\phi_{0}\left(w_{0}\right)+\sum_{p \in \mathcal{P}}\left(\phi_{p}\left(w_{0}, w_{p}\right)+\lambda^{T}\left(\bar{\Pi}_{p} w_{0}+\underline{\Pi}_{p} w_{p}\right)\right) .
$$

The first-order optimality conditions are given by:

$$
\begin{aligned}
\nabla_{w_{0}} \mathcal{L} & =0=\nabla_{w_{0}} \phi_{0}+\sum_{p \in \mathcal{P}}\left(\nabla_{w_{0}} \phi_{p}+\bar{\Pi}_{p}^{T} \lambda\right) \\
\nabla_{w_{p}} \mathcal{L} & =0=\nabla_{w_{p}} \phi_{p}+\underline{\Pi}_{p}^{T} \lambda, p \in \mathcal{P} \\
\nabla_{\lambda} \mathcal{L} & =0=\sum_{p \in \mathcal{P}}\left(\bar{\Pi}_{p} w_{0}+\underline{\Pi}_{p} w_{p}\right) .
\end{aligned}
$$

The linearization of these equations is simply,

$$
\begin{aligned}
W_{00} \Delta w_{0}+\sum_{p \in \mathcal{P}}\left(W_{0 p} \Delta w_{p}+\bar{\Pi}_{p}^{T} \Delta \lambda\right) & =-\nabla_{w_{0}} \mathcal{L} \\
W_{p 0} \Delta w_{0}+W_{p p} \Delta w_{p}+\underline{\Pi}_{p}^{T} \Delta \lambda & =-\nabla_{w_{p}} \mathcal{L}, p \in \mathcal{P} \\
\sum_{p \in \mathcal{P}}\left(\bar{\Pi}_{p} \Delta w_{0}+\underline{\Pi}_{p} \Delta w_{p}\right) & =-\nabla_{\lambda} \mathcal{L} .
\end{aligned}
$$

where $W_{00}:=\nabla_{w_{0}, w_{0}} \mathcal{L}, W_{p p}:=\nabla_{w_{p}, w_{p}} \mathcal{L}, W_{0 p}:=\nabla_{w_{0}, w_{p}} \mathcal{L}$, and $W_{p 0}:=\nabla_{w_{p}, w_{0}} \mathcal{L}$. This linear system 
can be expressed in matrix form as:

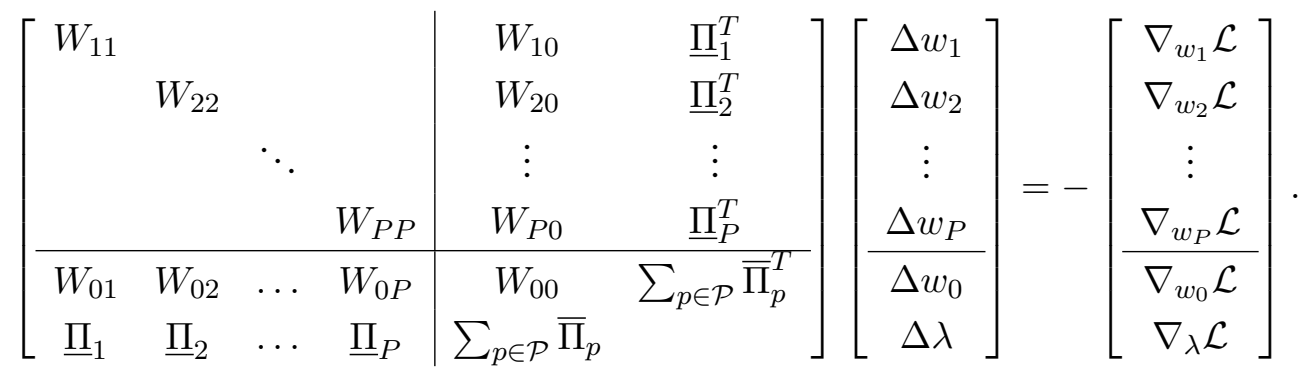

We thus have that the linear system is block-bordered diagonal and thus can be solved using a Schur complement decomposition approach [27, 29]. The Schur complement is of the same size as the dimension of $w_{0}$ and $\lambda$ and is often a dense matrix. This approach exhibits linear scaling in the number of scenarios if the number of coupling variables and constraints is of a few thousand variables and constraints $[49,27]$. In particular, the dense factorization of large Schur complement matrices dominates scaling in the scenario space. More sophisticated approaches exist to deal with large Schur matrices but these do not exhibit linear scaling [33, 8]. We have implemented the proposed parallel decomposition approach in the interior-point solver P IPS-NLP [10].

\subsection{Modeling SMOOs in PLASMO}

We use PLASMO to implement the SMOO problems. PLASMO (Platform for Scalable Modeling and Optimization) is a Julia-based modeling framework that facilitates the construction and analysis of structured optimization models. To do this, it leverages a hierarchical graph abstraction wherein nodes and edges can be associated with individual optimization models that are linked together [25]. Given a graph structure with models and connections, PLASMO can produce either a pure (flattened) optimization model to be solved using off-the-shelf optimization solvers such as IPOP T [44], or it can communicate graph structures to parallel solvers such as P IPS-NLP and thus enable decomposition. PLASMO leverages the basic algebraic modeling syntax and automatic differentiation capabilities of JuMP [32]. By using a graph representation, automatic differentiation can be performed separately on each model associated to a given node. Moreover, by using a graph abstraction we can create sophisticated operations on individual nodes. For instance, we can warm-start a large-scale problem by first finding solutions for individual nodes.

A standard two-stage stochastic NLP induces a two-level hierarchical graph where in the parent node is a model that contains global variables and constraints (first-stage) while the children nodes are connected to the parent and contain their own model with local variables and constraints (secondstage or recourse). In this case, each children node corresponds to the model of each scenario or partition. In the case of the stochastic NLP with linking constraints (3.25), we can still pose the problem as a two-level graph by adding the coupling variables and linking constraints to the parent node.

The code snippet shown in Figure 1 illustrates how to implement problem (C) in PLASMO. As can be seen, the individual scenario models are created and appended to the parent node on-the-fly to create a two-level graph structure. The structure is directly communicated to PIPS-NLP and thus 
Figure 1: Snippet of a nested CVaR implementation (Formulation (C)) in P LASMO

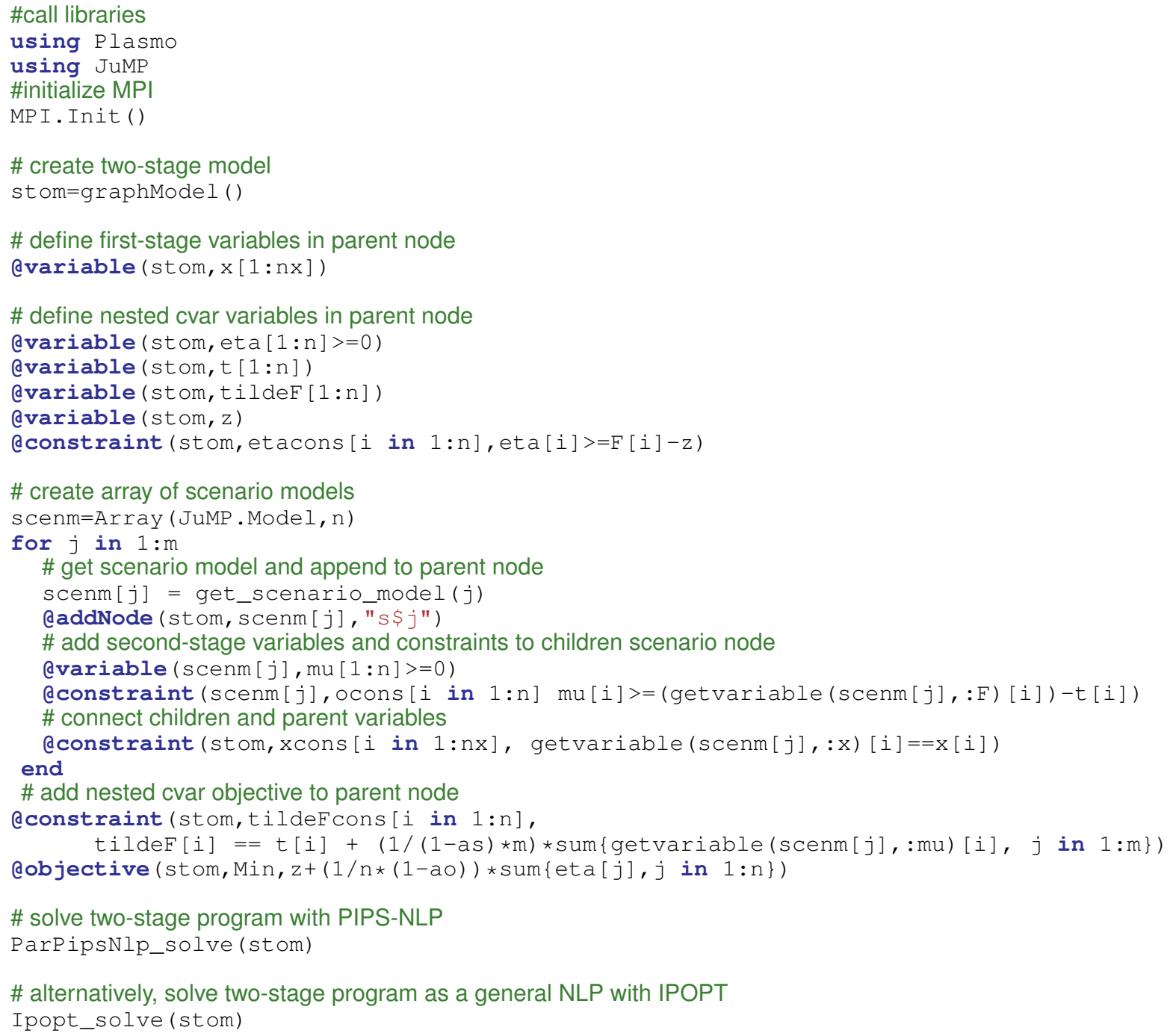

the solver can execute the parallel Schur decomposition approach previously discussed. Note also that, under this modeling framework, the user does not need to have any knowledge on parallel computing. From the snippet we also note that P LASMO can also create a general (unstructured) NLP to be solved by off-the-shelf solvers like IPOP T [44]. This allows the user to compare computational performance and evaluate, for instance, if parallelization actually pays off.

\section{CHP Case Study}

We consider the optimal design of a CHP system that provides electricity and thermal energy to a residential housing complex [17]. As with most building systems, electricity and hot water demands follow very different patterns and are out of phase, and this greatly complicates the design and operation of CHP systems [47]. Because of this, CHP systems often interact with the local utility company, which allows the system to sell electrical power surplus or to buy electricity when production of the 
CHP system is insufficient to meat the building demands [7]. This interaction is also essential because hot water is usually the limiting resource demand. Another complication in the design and operation is that demands vary strongly throughout the year due to seasonal effects.

The CHP system under study includes an internal combustion engine (ICE) and a thermal storage tank (ST). The ICE system under consideration uses natural gas to fuel the generator [46]. The thermal storage tank allows synchronization of electricity and thermal energy demands. For instance, it is possible to store the surplus of hot water during periods when electricity demand is far larger than hot water demands. The stored thermal energy can then be used in periods of high thermal demand and/or when it is not convenient to sell electricity to the utility plant [6]. The water is usually stored at high temperature to prevent the onset of pathogenic agents. Consequently, fresh water is often used to regulate the temperature of the delivered water to the residential buildings. The CHP system also requires a water purging system that allows to drain the excess hot water that can not be stored or consumed [45]. Purge water temperature must also be regulated before disposing of it. Water temperature regulation and storage management can thus lead to high water demands and are often in conflict with system economics. For instance, at certain times the CHP system might aggressively seek to sell electricity to the utility company but the thermal storage might already be at full capacity and thus there is an incentive to purge excess water. Thermal storage is also essential to prevent the ICE system from operating at partial loads, which has a strong effect on fuel efficiency and emissions. In particular, a CHP unit is most efficient at full load operation but, depending on real-time conditions on storage, utility prices, and demands; the CHP operator might seek to sacrifice fuel efficiency and increase emissions in order to improve economics [18, 39]. The size of the storage tank and the capacity of the CHP system together with operating policies thus play a critical role on the performance and trade-offs of the system.

\subsection{Optimal Design and Control Formulation}

We construct a simultaneous design and control model that seeks to trade-off water, emissions, and operational costs in CHP systems. The model incorporates energy and material balances of the CHP unit and the dynamics of the storage system. We also model the interactions with the utility company and quantify emissions and water use. The design seeks to mitigate variability in daily electricity and hot water demand profiles observed throughout the year. To do so, we model the daily demand profiles as scenarios. We begin our discussion by providing the equations describing a deterministic multiobjective optimization (MOO) formulation (for a single demand profile scenario) and we then describe extensions to the SMOO formulation.

\subsubsection{Electricity Demands and Grid Interaction}

The total electricity demand of the multi-building system (denoted as $W_{t}^{D}$ where $t$ is the time instant) is met using a fraction $\left(W_{t}^{C}\right)$ of the total electricity production of the CHP unit $\left(W_{t}^{C H P}\right)$. If the CHP system decides not to allocate all of the CHP generation to meet the demand, the system can buy electricity from the local utility company (this amount is denoted as $W_{t}^{B}$ ). The system can also decide to sell some fraction of the electricity produced in the CHP unit to the utility company (we denote 
this as $\left.W_{t}^{S}\right)$. This logic is modeled using the power balances

$$
\begin{gathered}
W_{t}^{D}=W_{t}^{C}+W_{t}^{B} \\
W_{t}^{C H P}=W_{t}^{C}+W_{t}^{S}
\end{gathered}
$$

These two balances imply the global balance:

$$
W_{t}^{D}=W_{t}^{C H P}-W_{t}^{S}+W_{t}^{B}
$$

\subsubsection{Hot Water Demands and Purge}

The demand of hot water $\left(G_{t}^{D}\right)$ is met using hot water from the storage $\left(G_{t}^{S T}\right)$ and cold water from the utility company $\left(G_{t}^{C W}\right)$ at ambient temperature $\left(T_{t}^{a m b}\right)$. The cold water is used for regulating the temperature of the hot water delivered to the buildings and to control the temperature of the purging system. The purge and demand temperature is given by $T^{D}$, which is typically required to be $50{ }^{\circ} \mathrm{C}$ [11]. This logic modeled using the following mass and energy balances:

$$
\begin{gathered}
G_{t}^{D}+G_{t}^{P}=G_{t}^{S T}+G_{t}^{C W} \\
\left(G_{t}^{D}+G_{t}^{P}\right) T^{D}=\left(G_{t}^{S T} T_{t}^{S T}\right)+\left(G_{t}^{C W} T_{t}^{a m b}\right)
\end{gathered}
$$

\subsubsection{Thermal Storage Dynamics}

The storage temperature $\left(T_{t}^{S T}\right)$ and volume level $\left(V_{t}^{S T}\right)$ depend on the inlet stream given by the hot water generated by the CHP unit $\left(G_{t}^{C H P}\right)$, and by the outlet stream from the storage $\left(G_{t}^{S T}\right)$ used for meeting the hot water demand of the building system. We also consider convective losses in the system, which are a function of the heat transfer area $\left(A_{t}^{S T}\right)$ and the ambient temperature $\left(T_{t}^{a m b}\right)$ [28]. The transfer area is dynamic, as it depends on the storage level. The storage system dynamics are captured using the following set of equations:

$$
\begin{gathered}
\rho\left(V_{t}^{S T}-V_{t-1}^{S T}\right)=G_{t}^{C H P}-G_{t}^{S T} \\
\rho c_{p}\left(V_{t}^{S T} T_{t}^{S T}+V_{t-1}^{S T} T_{t-1}^{S T}\right)=\left(G_{t}^{C H P} c_{p} T^{C H P}\right)-\left(G_{t}^{S T} c_{p} T_{t}^{S T}\right)-Q_{t}^{L} \\
Q_{t}^{L}=U A_{t}^{S T}\left(T_{t}^{S T}-T_{t}^{a m b}\right) \\
A_{t}^{S T}=6\left(V_{t}^{S T}\right)^{2 / 3}
\end{gathered}
$$

The water stored in the tank needs to satisfy a minimum temperature, which is usually set to $\underline{T}^{S T}=$ $70{ }^{\circ} \mathrm{C}$ :

$$
T_{t}^{S T} \geq \underline{T}^{S T}
$$

This temperature prevents proliferation of Legionella [30]. The storage volume level is constrained by the design capacity $V^{c a p}$ as:

$$
V_{t}^{S T} \leq V^{c a p}
$$




\subsubsection{Fuel Use and Partial Load Effects}

The thermal and electrical efficiencies (denoted as $\eta_{t}^{Q C H P}$ and $\eta_{t}^{W C H P}$ ) of the CHP unit are related to the production of heat and power and to the fuel flow as:

$$
\begin{gathered}
\eta_{t}^{Q C H P}=\frac{Q_{t}^{C H P}}{F_{t}^{C H P}} \\
\eta_{t}^{W C H P}=\frac{W_{t}^{C H P}}{F_{t}^{C H P}}
\end{gathered}
$$

The partial load $\left(P L_{t}\right)$ is defined as the ratio of total production of electrical energy $\left(W_{t}^{C H P}\right)$ and the CHP design capacity $\left(W^{c a p}\right)$ :

$$
P L_{t}=\frac{W_{t}^{C H P}}{W^{c a p}} .
$$

The effect of the partial load on the efficiency of the CHP unit is modeled using the equation proposed in [36]:

$$
\eta_{t}^{W C H P}=\left(0.324 P L_{t}^{2}+0.44 P L_{t}+0.235\right) \hat{\eta}^{W C H P}
$$

where $\hat{\eta}^{W C H P}$ is the maximum (nominal) system efficiency. Note that efficiency decreases in a highly nonlinear manner with the partial load and that consumption of fuel increases. The total fuel consumption $\left(F_{t}^{C H P}\right)$ can be met by natural gas $\left(N G_{t}\right)$ :

$$
F_{t}^{C H P}=N G_{t}
$$

The partial load also has a minimum limit in order to prevent system starvation effects:

$$
P L_{t} \geq \underline{P L} .
$$

The total hot water produced $\left(G_{t}^{C H P}\right)$ at temperature $\left(T^{C H P}\right)$ is related to the heat production as:

$$
Q_{t}^{C H P}=G_{t}^{C H P} c_{p}\left(T^{C H P}-T_{t}^{a m b}\right) .
$$

\subsubsection{Total Direct Emissions}

The total direct greenhouse gas emissions $(\mathrm{GH})$ are calculated for natural gas according to their corresponding emissions factor $\left(f_{G H}\right)$ :

$$
G H=\sum_{t=1}^{T} f_{G H}^{N G} \cdot N G_{t}
$$

where $T$ is the final time index (we consider a 24 hour horizon with time increments of one hour).

\subsubsection{Total Water Consumption}

The total water consumption (SW) accounts for the water used by the CHP unit to run the ICE and for the water used to regulate its temperature:

$$
S W=\sum_{t=1}^{T}\left(G_{t}^{C H P}+G_{t}^{C W}\right) .
$$




\subsubsection{Total Cost}

The total negative revenue (total cost) factors capital costs as well as income from sales of heat and power, the cost of operation and maintenance, cost of fuel, and purchase of power from the grid:

$$
T C=\operatorname{Cost}^{F}+\text { Cost }^{O M}+\text { Cost }^{\text {Fuel }}+\operatorname{Cost}^{P}-I^{W}-I^{H}
$$

The revenue of the CHP system is calculated considering the sales of electrical power and thermal energy. The power sales $\left(I^{W}\right)$ include the sales to the building system $\left(W_{t}^{C}\right)$ and to the utility company $\left(W_{t}^{S}\right)$. The price is set by a time-dependent tariff $\left(P E S_{t}\right)$ provided by the utility company. The total power sales are given by:

$$
I^{W}=\sum_{t=1}^{T} P E S_{t} \cdot\left(W_{t}^{C}+W_{t}^{S}\right) .
$$

The heat sales $\left(I^{H}\right)$ are given by the total production of heat in the CHP unit $\left(Q_{t}^{C H P}\right)$ minus the convective losses $\left(Q_{t}^{L}\right)$. The revenue of thermal energy production is determined by the heating price $(P H S)$ :

$$
I^{H}=P H S \cdot \sum_{t=1}^{T}\left(Q_{t}^{C H P}-Q_{t}^{L}\right) .
$$

The cost of maintenance of the CHP unit is based on the unitary cost of operation and maintenance associated to the total power production:

$$
\operatorname{Cost}^{O M}=\operatorname{UCost}^{O M} \sum_{t=1}^{T} W_{t}^{C H P} .
$$

The cost of fuel (Cost $\left.{ }^{\text {Fuel }}\right)$ is determined by prices of natural gas $(N G)$ :

$$
\text { Cost }^{\text {Fuel }}=\sum_{t=1}^{T} \operatorname{UCost}^{N G} N G_{t}
$$

The total cost of power purchased from the grid at price $\left(U \operatorname{Cost}_{t}^{P}\right)$ is given by:

$$
\operatorname{Cost}^{P}=\sum_{t=1}^{T}\left(\operatorname{CCost}_{t}^{P} W_{t}^{B}\right) .
$$

The capital (fixed) cost associated to the installation of the CHP system is given by:

$$
\operatorname{Cost}^{F}=\alpha^{S T} V^{c a p}+\alpha^{W} W^{c a p} \text {. }
$$

\subsubsection{Final MOO Representation}

The deterministic design and control problem is a MOO problem of the form:

$$
\begin{gathered}
\min (T C, G H, S W) \\
\text { s.t. }(4.30)-(4.56) .
\end{gathered}
$$

The design variables are the thermal storage design capacity $V^{S T}$ and the CHP design capacity $W^{c a p}$. The operational variables are flows, temperatures, storage volume levels, and so on. The objective functions are daily cost, emissions, and water usage. 


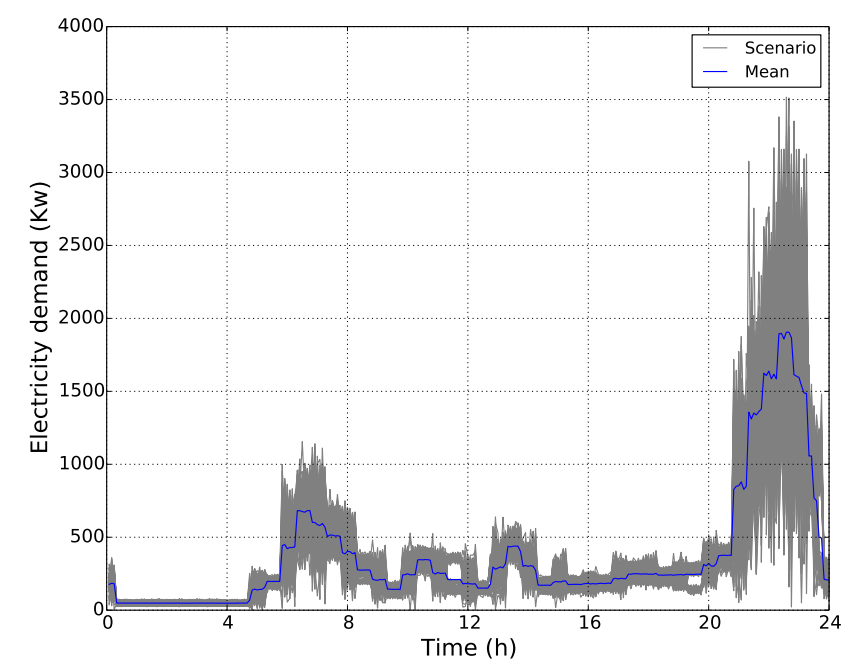

Figure 2: Daily electricity demand scenarios.

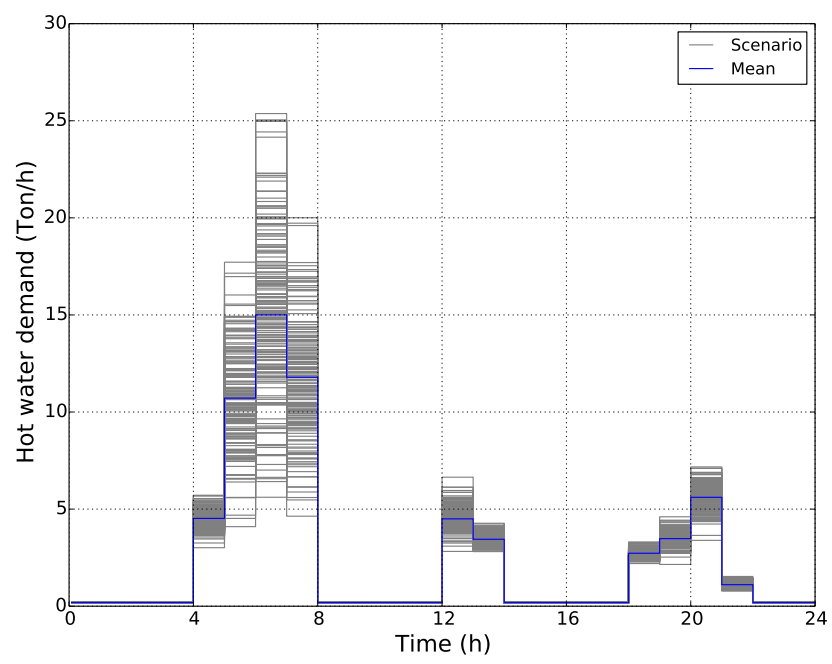

Figure 3: Daily hot water demand scenarios.

\subsection{SMOO Formulation and Compromise Solutions}

The SMOO formulation is obtained by considering multiple daily scenarios for the electrical and hot water demands $W_{t}^{D}$ and $G_{t}^{D}$ and of ambient temperatures $T_{t}^{a m b}$. To capture the variability of demands, we discretize the 24 hour time horizon using a 5 minute mesh. The design (first-stage) decision variables are the thermal storage design volume capacity $V^{S T}$ and the CHP design capacity $W^{c a p}$. The operation (resource) variables are flows, temperatures, storage volume levels, and so on. The random objective functions are $T C, G H, S W$ (which vary in each scenario).

We consider a residential housing complex comprising of 1,440 households and 7,632 inhabitants. We use an ICE with a maximum possible capacity for $W^{\text {cap }}$ of $200 \mathrm{~kW}$, efficiencies $\hat{\eta}^{W C H P}=37.25 \%$ and $\eta^{Q C H P}=47.5 \%$, and minimum partial load $\underline{P L}=0.35$. The maximum possible storage tank capacity $V^{c a p}$ is limited to $50 \mathrm{~m}^{3}$. The multi-building system is located in the State of Michoacan in 
Mexico (by the Pacific coast and near the mountains). The climatic conditions in this region are stable throughout the year. Real demand data for electricity and hot water for the buildings is presented in Figures 2 and 3. As can be seen, demands follow quasi-periodic patterns but are out of phase (hot water demands peak at the beginning of the day when the residents take showers while electricity demand peaks at night). Moreover, one can see that the there is a high variance of the demands at peak times. The electricity price from the utility company doubles during the time period of 5 p.m. and 10 p.m. The emission factor for natural gas $f_{G H}^{N G}$ is $380 \mathrm{~g} \mathrm{CO}_{2} / \mathrm{kWh}$ [26]. The thermal energy price $P H S$ is $0.015 \$ / \mathrm{kWh}$.

We scale all the objective functions using the utopia solutions. We use the nested CVaR formulations to identify different compromise solutions. The models were implemented on PLASMO [15] and solved with P IPS-NLP [44]. All scripts are available at http: / / zavalab.engr.wisc.edu/data.

Table 1: Compromise solutions for Formulations C and D

\begin{tabular}{|c|c|c|c|c|c|c|c|c|c|}
\hline \multicolumn{10}{|c|}{ Formulation C } \\
\hline$\alpha_{\mathcal{O}}$ & $\alpha_{\mathcal{S}}$ & $V^{c a p}\left(\mathrm{~m}^{3}\right)$ & $W^{c a p}(\mathrm{~kW})$ & $\overline{G H}$ (Ton) & $\overline{S W}$ (Ton) & $\overline{T C}(\$)$ & $G H^{\max }$ (Ton) & $S W^{\max }$ (Ton) & $T C^{\max }(\$)$ \\
\hline 0 & 0 & 22.55 & 113.68 & 2.41 & 77.011 & 671.40 & 2.79 & 88.555 & 861.72 \\
\hline 1 & 1 & 22.55 & 113.68 & 2.74 & 89.161 & 672.87 & 2.79 & 99.677 & 705.23 \\
\hline 1 & 0 & 49.94 & 113.68 & 2.62 & 85.014 & 596.21 & 2.79 & 88.528 & 769.05 \\
\hline 0 & 1 & 22.55 & 113.68 & 2.69 & 87.065 & 690.01 & 2.79 & 87.407 & 728.39 \\
\hline \multicolumn{10}{|c|}{ Formulation D } \\
\hline$\alpha_{\mathcal{S}}$ & $\alpha_{\mathcal{O}}$ & $V^{c a p}\left(\mathrm{~m}^{3}\right)$ & $W^{c a p}(\mathrm{~kW})$ & $\overline{G H}$ (Ton) & $\overline{S W}$ (Ton) & $\overline{T C}(\$)$ & $G H^{\max }$ (Ton) & $S W^{\max }$ (Ton) & $T C^{\max }(\$)$ \\
\hline 1 & 0 & 22.55 & 113.68 & 2.62 & 84.892 & 707.86 & 2.79 & 89.301 & 861.67 \\
\hline 0 & 1 & 22.55 & 113.68 & 2.62 & 85.522 & 602.24 & 2.79 & 99.426 & 705.65 \\
\hline
\end{tabular}

The compromise solutions obtained with Formulation $C$ and D are shown in Table 1. Here, we summarize the mean and worst-case values for the different objectives. The entire set of costs, water, and emissions along with their corresponding utopia points are shown in Figures 4-9. As can be seen, the average compromise solution (obtained by minimizing $\langle\langle\mathbf{F}(x)\rangle\rangle_{\alpha_{\mathcal{O}}=0, \alpha_{\mathcal{S}}=0}$ ) shows a large spread in the objective space. This spread is manifested in a large difference between the mean and worstcase objetives. For the cost we have $\$ 671.40$ and $\$ 861.72$ (a difference of $28 \%$ ), for the emissions we have 2.41 and 2.79 Ton (a difference of 15\%), and for water usage we have 77.01 Ton and 88.55 Ton (a difference of $15 \%$ ). The worst-case compromise solution (obtained by minimizing $\langle\langle\mathbf{F}(x)\rangle\rangle_{\alpha_{\mathcal{O}}=1, \alpha_{\mathcal{S}}=1}$ ) reduces the relative differences to $4.9 \%, 2 \%$, and $11.8 \%$. The reduction in the variability can be clearly visualized by comparing Figures 4 and 5. Interestingly, the optimal size for the storage and the CHP system is the same for both compromise solutions. This indicates that the operational policy places a critical role in shaping the distribution of the objectives. We can also observe clear changes in the distribution of the objectives by comparing the compromise solutions obtained by minimizing the worst-case of the average scenario objectives (obtained by minimizing $\left\langle\langle\mathbf{F}(x)\rangle_{\alpha_{\mathcal{O}}=1, \alpha_{\mathcal{S}}=0}\right.$ ) and obtained by minimizing the average of the worst-case scenario objectives (obtained by minimizing $\left.\langle\langle\mathbf{F}(x)\rangle\rangle_{\alpha_{\mathcal{O}}=0, \alpha_{\mathcal{S}}=1}\right)$. In particular, for the first case we see that the objectives form three clusters with a large variance in cost while in the second case we only observe two clusters with a smaller variance in cost. 


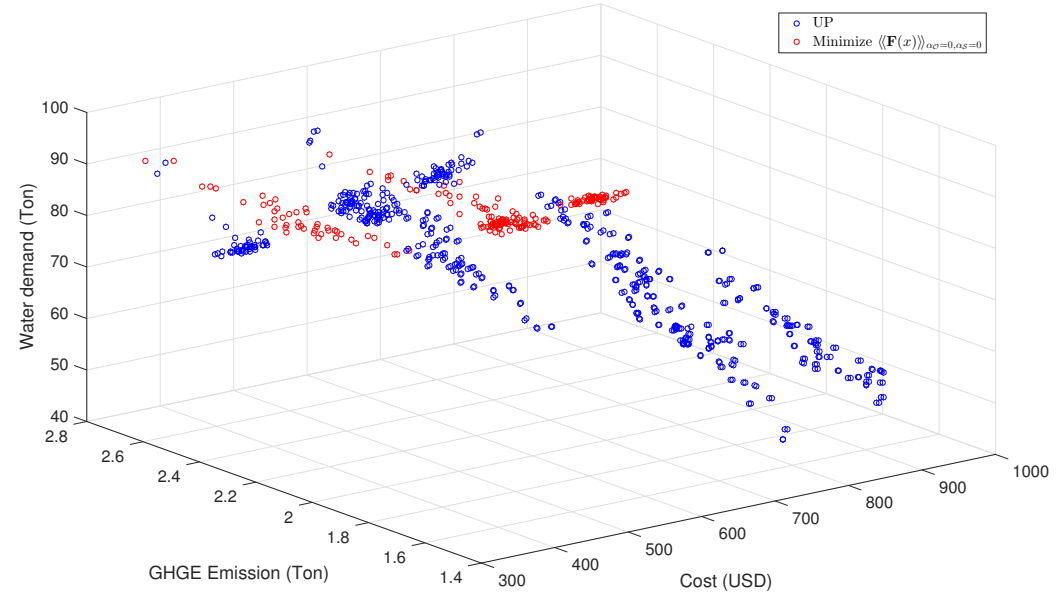

Figure 4: Utopia and compromise solution for $\min _{x \in \mathcal{X}}\langle\langle\mathbf{F}(x)\rangle\rangle_{\alpha_{\mathcal{O}}=0, \alpha_{\mathcal{S}}=0}$.

Interestingly, the only solution that has different design capacities is the one that minimizes the worst-case of the average objectives. This solution increases the capacity of the storage tank by $100 \%$ compared to the other designs. This allows the system to significantly reduce average cost (by 11\%) compared to the closest design. This can be explained by the fact that a larger storage system allows the system to produce and sell more electricity to the utility company during periods of high prices. On the other hand, this design has a larger worst-case cost than that obtained by other designs. In other words, this design can be seen as a central solution between the average and worst-case compromise solutions. We also note that the optimal capacities for the rest of the compromise solutions do not hit the upper bounds $V^{c a p}$ and $W^{c a p}$, which indicates that the design seeks to optimally balance sales and purchases of electricity from the utility. In other words, it is not optimal to build a large CHP system to fully satisfy the electricity demands of the residential complex. Such a large CHP design would force the system to operate at partial loads most of the time and will thus be inefficient from a fuel usage standpoint. Moreover, operating such a large CHP system will also waste significant amounts of water as the peak water demands do not match the peak demands of electricity.

In Table 1 and Figures 8 and 9 we compare the compromise solutions obtained by minimizing the worst-case over the average of the three objectives (obtained with $\left\langle\langle\mathbf{F}(x)\rangle_{\alpha_{\mathcal{S}}=0, \alpha_{\mathcal{O}}=1}\right.$ ) and by minimizing the average over the worst-case of the three objectives (obtained with $\langle\langle\mathbf{F}(x)\rangle\rangle_{\alpha_{\mathcal{S}}=1, \alpha_{\mathcal{O}}=0}$ ). These solutions also obtain the same design as the solutions obtained with Formulation $\mathrm{C}$. The most interesting behavior is obtained by minimizing $\langle\langle\mathbf{F}(x)\rangle\rangle_{\alpha_{\mathcal{S}}=0, \alpha_{\mathcal{O}}=1}$. Under this solution, the objectives cluster around a plane with large water usage variance but narrow cost, which can be confirmed by verifying average and worst-case values in Table 1. This formulation has the interesting property that it first obtains the worst-case objective for each scenario and then it averages them. This solution can thus be interpreted as an average of the worst-case objectives. We again highlight that the proposed framework allows practitioners to obtain solutions with different interpretations. This can 


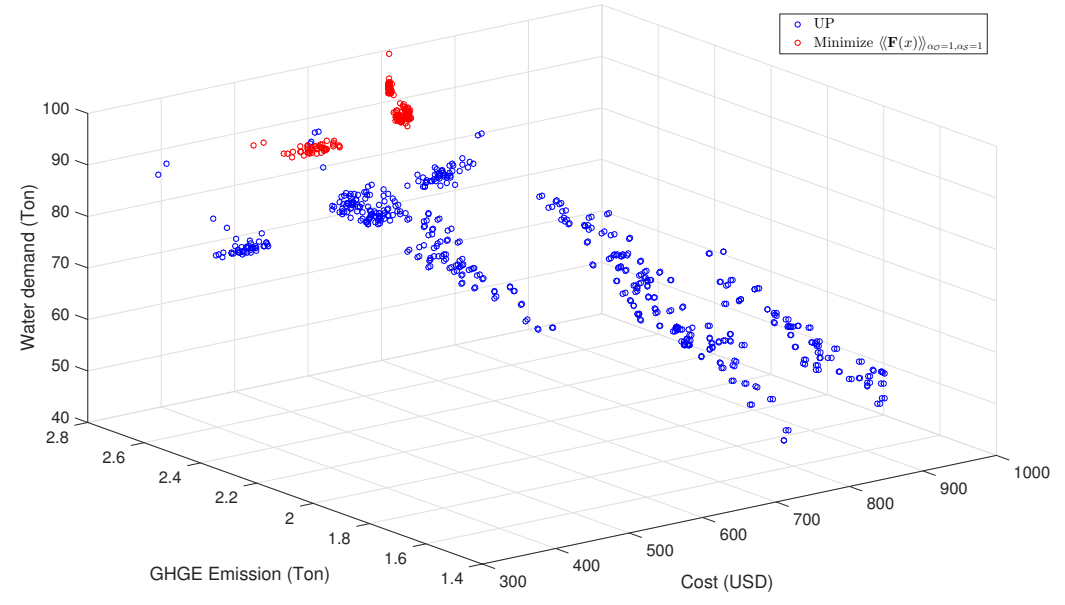

Figure 5: Utopia and compromise solution for $\min _{x \in \mathcal{X}}\langle\langle\mathbf{F}(x)\rangle\rangle_{\alpha_{\mathcal{O}}=1, \alpha_{\mathcal{S}}=1}$.

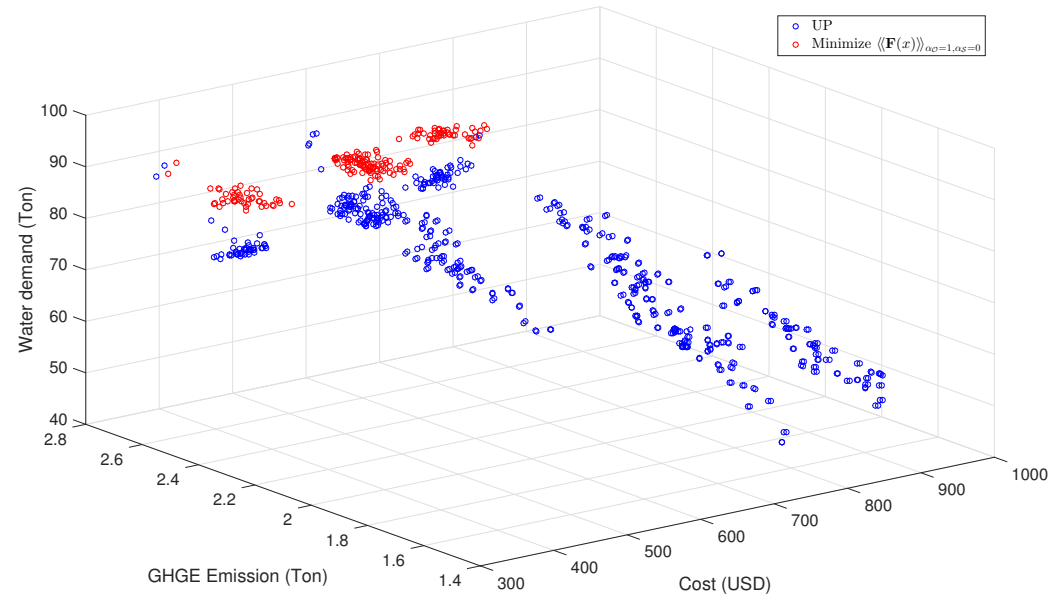

Figure 6: Utopia and compromise solution for $\min _{x \in \mathcal{X}}\langle\langle\mathbf{F}(x)\rangle\rangle_{\alpha_{\mathcal{O}}=1, \alpha \mathcal{S}=0}$. 


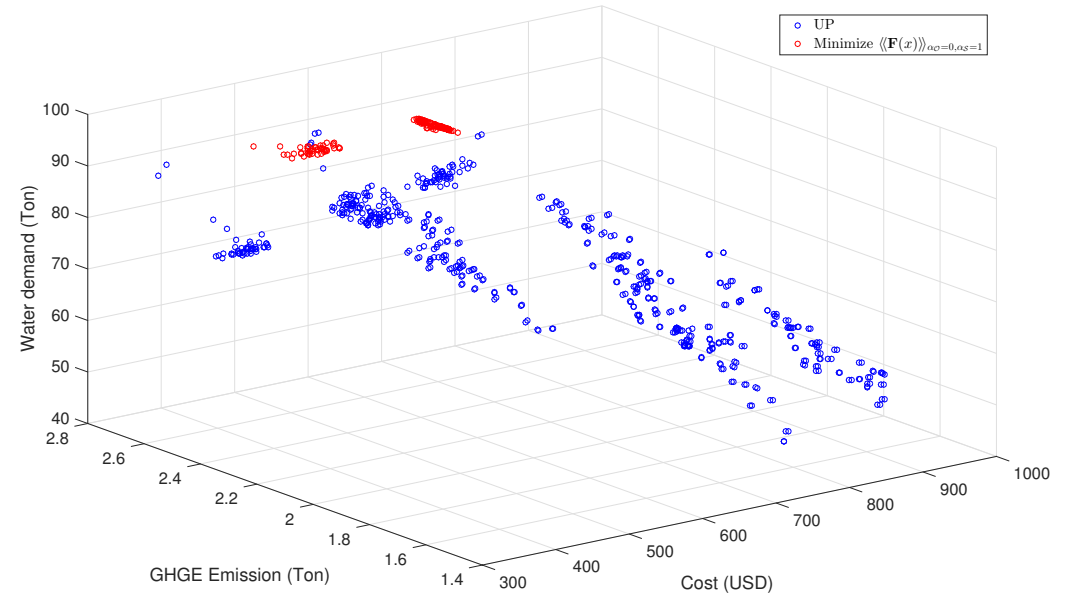

Figure 7: Utopia and compromise solution for $\min _{x \in \mathcal{X}}\left\langle\langle\mathbf{F}(x)\rangle_{\alpha_{\mathcal{O}}=0, \alpha_{\mathcal{S}}=1}\right.$.

allow decision-makers to identify designs and operational policies that satisfy their individual priorities. Of course, there is some ambiguity associated to the selection of the nested CVaR parameters but we note that the decision space has been significantly narrowed down from the full scenario and objective space to a handful of solutions. Moreover, we highlight that the minimization of the nested $\mathrm{CVaR}$ metric allows us to compute compromise solutions without having to compute entire Pareto sets.

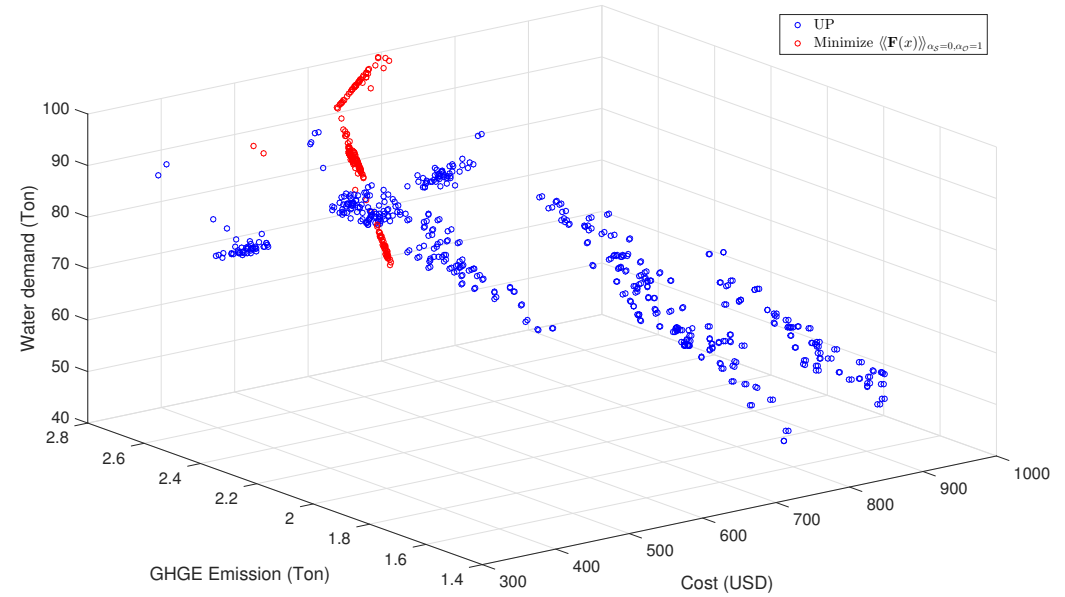

Figure 8: Utopia and compromise solution for $\min _{x \in \mathcal{X}}\langle\langle\mathbf{F}(x)\rangle\rangle_{\alpha_{\mathcal{S}}=0, \alpha_{\mathcal{O}}=1}$.

\subsection{Computational Performance}

We now discuss computational performance of the framework. Formulation (C) of the CHP problem results in a two-level graph model with 13 variables, 4 equality constraints, and 3 inequality constraints in the parent node and with 5776 variables, 4909 equality constraints, and 868 inequality 


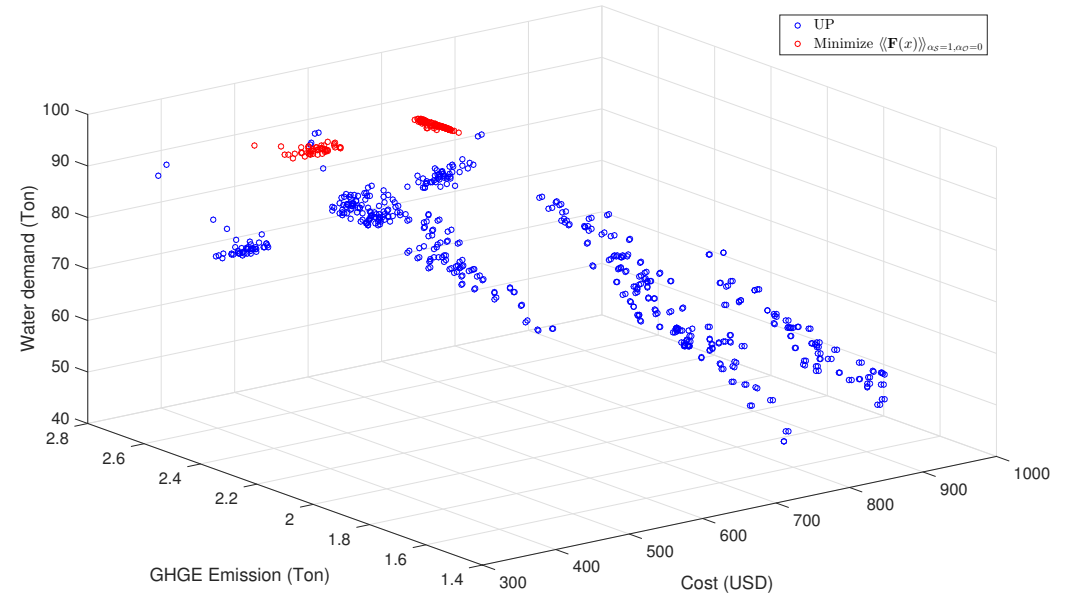

Figure 9: Utopia and compromise solution for $\min _{x \in \mathcal{X}}\langle\langle\mathbf{F}(x)\rangle\rangle_{\alpha_{\mathcal{S}}=1, \alpha_{\mathcal{O}}=0}$.

constraints per scenario (children) node. The problem under study has 188 scenarios which gives rise a total of 1,085,901 variables, 922,896 equality constraints, and 163,187 inequality constraints. We solve this problem using P IPS-NLP using a Schur decomposition approach on a multi-core shared memory environment. We compare performance against the full-space solution with IPOPT performed on a single core. Schur decomposition is not used in IPOPT (a direct sparse factorization is used). We perform this comparison to evaluate how well does a full-space sparse factorization performs against parallel Schur decomposition.

The computational results are summarized in Table 2. As can be seen, for the $\alpha_{\mathcal{O}}=0, \alpha_{\mathcal{S}}=0$ case, P IPS-NLP is able to reduce the computational times from 3,894 seconds (over an hour) to 378 seconds (6.3 minutes) using 20 cores. The solution time of IPOPT is 1,167 seconds (19.4 minutes). We thus can see that the direct factorization used in IPOPT is fairly competitive but PIPS-NLP is still significantly faster and can in principle be accelerated further by using more cores. We also observe that the number of iterations is comparable for both solvers (around 300 iterations). In the $\alpha_{\mathcal{O}}=1, \alpha_{\mathcal{S}}=1$ case we see a significant increase in the number of iterations (to over 800 ). We can thus see that nested CVaR settings can have a significant effect on performance. We attribute this to the fact that the worst-case solutions lead to more degeneracy in the solution in which the different scenarios are nearly indistinguishable (this can be seen from the tight scenario clusters shown in Figure 5). In this case, however, we still perceive a significant acceleration using P IP S-NLP. The solution times are reduced from 12,627 seconds ( 3.5 hours) to 1,439 seconds (23.98 minutes). The solution time obtained with IPOP T is 3,959 seconds (66 minutes). We thus see that the proposed decomposition framework provides important savings in computing time and can handle large problems. 
Table 2: Computational performance of PIPS-NLP and IPOPT on Formulation C.

\begin{tabular}{cccc|cc|cc|cc|cc}
\multicolumn{4}{c}{ P IPS (20 Cores) } & \multicolumn{2}{c}{ P IPS (10 Cores) } & \multicolumn{2}{c}{ P IPS (5 Cores) } & \multicolumn{2}{c}{ P IPS (1 Core) } & \multicolumn{2}{c}{ IP OP (1 Core) } \\
\hline$\alpha_{\mathcal{O}}$ & $\alpha_{\mathcal{S}}$ & Time(s) & Iter & Time(s) & Iter & Time(s) & Iter & Time $(\mathrm{s})$ & Iter & Time (s) & Iter \\
\hline 0 & 0 & 378 & 313 & 547 & 313 & 1,022 & 313 & 3,894 & 313 & 1,167 & 325 \\
1 & 1 & 1,439 & 871 & 1,957 & 848 & 3,065 & 843 & 12,627 & 838 & 3,959 & 853
\end{tabular}

\section{Conclusions}

We presented a scalable computing framework for the solution stochastic multiobjective optimization problems. Our framework uses a nested conditional value-at-risk (nCVaR) objective to find compromise solutions among conflicting random objectives. We prove that the associated $\mathrm{nCVaR}$ minimization problems can be cast as standard stochastic programming problems with expected value constraints. We also show that these problems can be implemented in a highly modular fashion using PLASMO (a Julia-based structured modeling framework) and can be solved efficiently using the parallel solver P IPS-NLP. We show that the framework can be used to compute a rich set of compromise solutions that seek to balance objectives and performance in different scenarios. As part of future work, we will consider extensions to model a wider range of problems that involve multiple decision layers. Such problems arise, for instance, when stakeholders disagree on which compromise solution should be used (i.e., which combination of probability levels $\alpha_{\mathcal{O}}$ and $\alpha_{\mathcal{S}}$ should be used).

\section{Acknowledgments}

We acknowledge funding from the U.S. Department of Energy under grant DE-SC0014114 and from the National Science Foundation under grant CBET-1604374.

\section{References}

[1] F. B. Abdelaziz. Solution approaches for the multiobjective stochastic programming. European Journal of Operational Research, 216(1):1-16, 2012.

[2] F. B. Abdelaziz, B. Aouni, and R. El Fayedh. Multi-objective stochastic programming for portfolio selection. European Journal of Operational Research, 177(3):1811-1823, 2007.

[3] B. Armbruster and J. Luedtke. Models and formulations for multivariate dominanceconstrained stochastic programs. IIE Transactions, 47(1):1-14, 2015.

[4] B. Armbruster and J. Luedtke. Models and formulations for multivariate dominanceconstrained stochastic programs. IIE Transactions, 47(1):1-14, 2015. 
[5] F. F. Baesler and J. A. Sepúlveda. Multi-objective simulation optimization for a cancer treatment center. In Proceedings of the 2001 Winter Simulation Conference, volume 2, pages 1405-1411. IEEE, 2001.

[6] E. S. Barbieri, F. Melino, and M. Morini. Influence of the thermal energy storage on the profitability of micro-CHP systems for residential building applications. Applied Energy, 97:714-722, 2012.

[7] E. Bompard and T. Huang. Control of interconnected networks. In Monitoring, Control and Protection of Interconnected Power Systems, pages 195-213. Springer, 2014.

[8] Y. Cao, C. D. Laird, and V. M. Zavala. Clustering-based preconditioning for stochastic programs. Computational Optimization and Applications, 64(2):379-406, 2016.

[9] C. Chang and W. Fu. Stochastic multiobjective generation dispatch of combined heat and power systems. IEE Proceedings-Generation, Transmission and Distribution, 145(5):583-591, 1998.

[10] N.-Y. Chiang and V. M. Zavala. An inertia-free filter line-search algorithm for large-scale nonlinear programming. Computational Optimization and Applications, 64(2):327-354, 2016.

[11] T. Collin, S. Jeffery, and C. Reid. Bath-water scalds in children and thermostatic mixer valves. Burns, 32(7):909-912, 2006.

[12] D. Dentcheva and A. Ruszczynski. Optimization with stochastic dominance constraints. SIAM Journal on Optimization, 14(2):548-566, 2003.

[13] D. Dentcheva and A. Ruszczyński. Optimization with multivariate stochastic dominance constraints. Mathematical Programming, 117(1-2):111-127, 2009.

[14] A. W. Dowling, G. Ruiz-Mercado, and V. M. Zavala. A framework for multi-stakeholder decision-making and conflict resolution. Computers E Chemical Engineering, 90:136-150, 2016.

[15] I. Dunning, J. Huchette, and M. Lubin. JuMP: A modeling language for mathematical optimization. arXiv preprint arXiv:1508.01982, 2015.

[16] M. C. Fonseca, Á. García-Sánchez, M. Ortega-Mier, and F. Saldanha-da Gama. A stochastic biobjective location model for strategic reverse logistics. Top, 18(1):158-184, 2010.

[17] L. F. Fuentes-Cortés, Y. Ma, J. M. Ponce-Ortega, G. Ruiz-Mercado, and V. M. Zavala. Valuation of water and emissions in energy systems. Applied Energy, 2016.

[18] P. Ghadimi, S. Kara, and B. Kornfeld. The optimal selection of on-site chp systems through integrated sizing and operational strategy. Applied Energy, 126:38-46, 2014.

[19] W. J. Gutjahr and A. Pichler. Stochastic multi-objective optimization: a survey on non-scalarizing methods. Annals of Operations Research, 236(2):475-499, 2016. 
[20] W. J. Gutjahr and P. Reiter. Bi-objective project portfolio selection and staff assignment under uncertainty. Optimization, 59(3):417-445, 2010.

[21] H. Hassan-Pour, M. Mosadegh-Khah, and R. Tavakkoli-Moghaddam. Solving a multi-objective multi-depot stochastic location-routing problem by a hybrid simulated annealing algorithm. Proceedings of the Institution of Mechanical Engineers, Part B: Journal of Engineering Manufacture, 223(8):1045-1054, 2009.

[22] M. Hennen, S. Postels, P. Voll, M. Lampe, and A. Bardow. Multi-objective synthesis of energy systems: Efficient identification of design trade-offs. Computers $\mathcal{E}$ Chemical Engineering, 97:283293, 2017.

[23] J. Hu, T. Homem-de Mello, and S. Mehrotra. Stochastically weighted stochastic dominance concepts with an application in capital budgeting. European Journal of Operational Research, 232(3):572-583, 2014.

[24] J. Hu and S. Mehrotra. Robust and stochastically weighted multiobjective optimization models and reformulations. Operations research, 60(4):936-953, 2012.

[25] J. Jalving, S. Abhyankar, K. Kim, M. Hereld, and V. M. Zavala. A graph-based computational framework for simulation and optimization of coupled infrastructure networks. Under Review, 2016.

[26] P. J. Jørgensen. Biogas-Green Energy: Process, Design, Energy Supply, Environment. Researcher for a Day, 2009.

[27] J. Kang, N. Chiang, C. D. Laird, and V. M. Zavala. Nonlinear programming strategies on highperformance computers. In Proc. of the IEEE Conference on Decision and Control, Osaka, Japan, 2015.

[28] A. Kar and K. Al-Dossary. Thermal performances of water heaters in series. Applied Energy, 52(1):47-53, 1995.

[29] C. D. Laird and L. T. Biegler. Large-scale nonlinear programming for multi-scenario optimization. In Modeling, simulation and optimization of complex processes, pages 323-336. Springer, 2008.

[30] E. Leoni, G. De Luca, P. Legnani, R. Sacchetti, S. Stampi, and F. Zanetti. Legionella waterline colonization: detection of legionella species in domestic, hotel and hospital hot water systems. Journal of Applied Microbiology, 98(2):373-379, 2005.

[31] F. Logist, B. Houska, M. Diehl, and J. F. Van Impe. Robust multi-objective optimal control of uncertain (bio)chemical processes. Chemical Engineering Science, 66(20):4670-4682, 2011.

[32] M. Lubin and I. Dunning. Computing in operations research using Julia. INFORMS Journal on Computing, 27(2):238-248, 2015. 
[33] M. Lubin, C. G. Petra, and M. Anitescu. The parallel solution of dense saddle-point linear systems arising in stochastic programming. Optimization Methods and Software, 27(4-5):845-864, 2012.

[34] A. Messac, A. Ismail-Yahaya, and C. A. Mattson. The normalized normal constraint method for generating the pareto frontier. Structural and multidisciplinary optimization, 25(2):86-98, 2003.

[35] K. Miettinen. Nonlinear multiobjective optimization, volume 12 of international series in operations research and management science, 1999.

[36] H. Onovwiona and V. Ugursal. Residential cogeneration systems: review of the current technology. Renewable and Sustainable Energy Reviews, 10(5):389-431, 2006.

[37] G. Ostrovsky, N. Ziyatdinov, and T. Lapteva. Optimal design of chemical processes with chance constraints. Computers E Chemical Engineering, 59:74-88, 2013.

[38] K. Pavlikov and S. Uryasev. Cvar norm and applications in optimization. Optimization Letters, 8(7):1999-2020, 2014.

[39] C. Schaumburg-Müller. A partial load model for a local combined heat and power plant. In Proceedings of the 1st Nordic optimization symposium, Copenhagen, 2006.

[40] P. Schütz, A. Tomasgard, and S. Ahmed. Supply chain design under uncertainty using sample average approximation and dual decomposition. European Journal of Operational Research, 199(2):409-419, 2009.

[41] K. Sindhya, V. Ojalehto, J. Savolainen, H. Niemistö, J. Hakanen, and K. Miettinen. Coupling dynamic simulation and interactive multiobjective optimization for complex problems: An aprosnimbus case study. Expert Systems with Applications, 41(5):2546-2558, 2014.

[42] A. Syberfeldt, A. Ng, R. I. John, and P. Moore. Multi-objective evolutionary simulationoptimisation of a real-world manufacturing problem. Robotics and Computer-Integrated Manufacturing, 25(6):926-931, 2009.

[43] M. Vallerio, J. Hufkens, J. Van Impe, and F. Logist. An interactive decision-support system for multi-objective optimization of nonlinear dynamic processes with uncertainty. Expert Systems with Applications, 42(21):7710-7731, 2015.

[44] A. Wächter and L. T. Biegler. On the implementation of an interior-point filter line-search algorithm for large-scale nonlinear programming. Mathematical Programming, 106(1):25-57, 2006.

[45] J. Wiehagen and J. Sikora. Performance comparison of residential hot water systems. NAHB Research Center, Upper Marlboro, Maryland, NREL, 2003.

[46] L. Yingjian, Q. Qi, H. Xiangzhu, and L. Jiezhi. Energy balance and efficiency analysis for power generation in internal combustion engine sets using biogas. Sustainable Energy Technologies and Assessments, 6:25-33, 2014. 
[47] Y. G. Yohanis, J. D. Mondol, A. Wright, and B. Norton. Real-life energy use in the UK: How occupancy and dwelling characteristics affect domestic electricity use. Energy and Buildings, 40(6):1053-1059, 2008.

[48] V. M. Zavala and A. Flores-Tlacuahuac. Stability of multiobjective predictive control: A utopiatracking approach. Automatica, 48(10):2627-2632, 2012.

[49] V. M. Zavala, C. D. Laird, and L. T. Biegler. Interior-point decomposition approaches for parallel solution of large-scale nonlinear parameter estimation problems. Chemical Engineering Science, 63(19):4834-4845, 2008. 